\title{
Arp2/3 inactivation causes intervertebral disc and cartilage degeneration with dysregulated TonEBP-mediated osmoadaptation
}

Steven Tessier, ${ }^{1}$ Alexandra C. Doolittle, ${ }^{1,2}$ Kimheak Sao, ${ }^{1,2}$ Jeremy D. Rotty, ${ }^{3,4,5}$ James E. Bear, ${ }^{4,5}$ Veronica Ulici, ${ }^{6,7}$ Richard F. Loeser, ${ }^{6,7}$ Irving M. Shapiro,, ${ }^{1,2}$ Brian O. Diekman, ${ }^{7,8}$ and Makarand V. Risbud ${ }^{1,2}$

${ }^{1}$ Department of Orthopaedic Surgery, Sidney Kimmel Medical College, ${ }^{2}$ Graduate Program in Cell Biology and Regenerative Medicine, Jefferson College of Life Sciences, Thomas Jefferson University, Philadelphia, Pennsylvania, USA. ${ }^{3}$ Department of Biochemistry, Uniformed Services University of the Health Sciences, Bethesda, Maryland, USA. ${ }^{4}$ Department of Cell Biology and Physiology, ${ }^{5} U N C$ Lineberger Comprehensive Cancer Center, ${ }^{6}$ Thurston Arthritis Research Center, and 'Division of Rheumatology, Allergy, and Immunology, Department of Medicine, School of Medicine, University of North Carolina at Chapel Hill, Chapel Hill, North Carolina, USA. ${ }^{8}$ Joint Department of Biomedical Engineering, University of North Carolina at Chapel Hill and North Carolina State University, Chapel Hill and Raleigh, North Carolina, USA.

Extracellular matrix and osmolarity influence the development and homeostasis of skeletal tissues through Rho GTPase-mediated alteration of the actin cytoskeleton. This study investigated whether the actin-branching Arp2/3 complex, a downstream effector of the Rho GTPases Cdc42 and Rac1, plays a critical role in maintaining the health of matrix-rich and osmotically loaded intervertebral discs and cartilage. Mice with constitutive intervertebral disc- and cartilage-specific deletion of the critical Arp2/3 subunit Arpc2 (Col2-Cre; Arpc2 ${ }^{\text {fl/fl }}$ ) developed chondrodysplasia and spinal defects. Since these mice did not survive to adulthood, we generated mice with inducible Arpc2 deletion in disc and cartilage (Acan-CreER ${ }^{\mathrm{T} 2}$; Arpc2 ${ }^{\mathrm{fl} / \mathrm{fl}}$ ). Inactivation of Arp2/3 at skeletal maturity resulted in growth plate closure, loss of proteoglycan content in articular cartilage, and degenerative changes in the intervertebral disc at 1 year of age. Chondrocytes with Arpc2 deletion showed compromised cell spreading on both collagen and fibronectin. Pharmacological inhibition of Cdc42 and Arp2/3 prevented the osmoadaptive transcription factor TonEBP/NFAT5 from recruiting cofactors in response to a hyperosmolarity challenge. Together, these findings suggest that Arp2/3 plays a critical role in cartilaginous tissues through the regulation of cell-extracellular matrix interactions and modulation of TonEBP-mediated osmoadaptation.

Conflict of interest: The authors have declared that no conflict of interest exists.

Copyright: () 2020, American Society for Clinical Investigation.

Submitted: June 27, 2019

Accepted: January 15, 2020

Published: February 27, 2020

Reference information: /CI Insight. 2020;5(4):e131382.

https://doi.org/10.1172/jici.

insight.131382.

\section{Introduction}

Arp2/3 is an evolutionarily conserved protein complex comprising 7 subunits that assemble to regulate the actin cytoskeleton $(1,2)$. Canonically, the Arp2/3 complex is widely known for its role as an actin nucleator, whereby it polymerizes the formation of new actin filaments from preexisting filaments, forming a y-shaped junction with a branch angle of approximately $70^{\circ}(3,4)$. This process, called dendritic nucleation, generates branched-actin networks essential for diverse cellular functions, including lamellipodia formation, cellextracellular matrix (cell-ECM) interactions, and osmoadaptation $(5,6)$. Arp2/3 is spatially and temporally governed by a host of regulatory molecules, including members of the Wiskott-Aldrich Syndrome protein (WASp) and WAVE/SCAR superfamilies, which function as nucleation promoting factors (NPFs). These NPFs operate downstream of the small Rho GTPases Cdc42 and Rac1 (7-9). Consequently, Cdc42 and Rac1 have emerged as critical regulators of Arp2/3-mediated actin remodeling through their activation of NPFs.

Conditional disruption of $\mathrm{Cdc} 42$ and Rac1 in mice has resulted in skeletal phenotypes characterized by dwarfism and severely disorganized growth plates (10-12). These phenotypes are consistent with in vitro experiments that established a role for $\mathrm{Cdc} 42$ and Rac1 in controlling the rate of chondrocyte proliferation, hypertrophic differentiation, and apoptosis (13). Moreover, chondrodysplasia was observed in mice with 
cartilage-specific loss of $\beta 1$ integrin, an adhesion receptor involved in the activation of Rho GTPases, as well as in mice with loss of integrin-linked kinase, an important intracellular effector of cell-ECM interactions (14-16). Likewise, loss of $\beta 1$ integrin in early limb mesenchyme showed structural defects in articular cartilage characterized by accelerated terminal differentiation of chondrocytes with associated altered cell shape and a disrupted actin cytoskeleton (17). Since chondrocyte interactions with the ECM are primarily mediated by integrins, these phenotypes are largely attributed to impaired cell-ECM interactions. Importantly, previous reports have shown that Arp2/3 components associate with integrin focal adhesions (18), where they form hybrid complexes with vinculin to form an anchoring module $(19,20)$. The notion that Arp2/3 activation is tightly coupled to integrin-mediated adhesion and Rho GTPase signaling led us to hypothesize that compromised Arp2/3 function might play a key role in these mutant mice.

Previous studies have also shown that osmotic stress induces activation of Cdc42 and Rac1, triggering Arp2/3 translocation to the cell cortex (21). Correspondingly, impaired volume control and increased cell death have been reported in Arp2/3-depleted fibroblasts challenged by hyperosmolarity, suggesting a compromised osmoadaptive response (5). It is important to note that tonicity-responsive enhancer binding protein (TonEBP, also known as NFAT5 or OREBP), an osmoregulatory transcription factor, is expressed by chondrocytes and intervertebral disc cells to maintain intracellular osmotic balance in their proteoglycan-rich and hyperosmolar extracellular environment (22-25). We have previously shown that TonEBP supports nucleus pulposus (NP) cell survival under hyperosmotic conditions by regulating the expression of key survival molecules such as COX-2 and Hsp70, as well as osmoadaptive genes including taurine transporter (TauT), sodium/myo-inositol cotransporter (SMIT), and aldose reductase (AR) (24, 26, 27). These osmoadaptive genes encode proteins involved in the intracellular import and synthesis of organic, nonionic osmolytes (28). Furthermore, TonEBP deficiency has been shown to delay spinal development and accelerate age-related intervertebral disc degeneration underscored by altered expression of actin cytoskeleton-related genes, including Arp2/3 subunits $(29,30)$. Noting the importance of osmoadaptation in cartilage and the intervertebral disc, we asked whether Arp2/3 regulates the activity of TonEBP.

Employing 2 conditional KO mouse models, our studies provide evidence that Arp2/3 deficiency causes severe growth plate defects in both vertebrae and the hind limb, along with loss of sulfated glycosaminoglycan content in articular cartilage. These phenotypes are associated with impaired chondrocyte cell-ECM interactions. We also provide morphologic and molecular evidence that conditional Arp2/3 loss results in intervertebral disc defects shortly after birth and later in adulthood. For the first time to our knowledge, in vitro experiments using primary NP cells demonstrate that Arp2/3 and Cdc42, but not Rac1, control TonEBP activity, lending mechanistic insights into the role of Arp2/3 in skeletal physiology.

\section{Results}

Inactivation of Arp2/3 in cartilage and disc causes embryonic and early postnatal lethality, dwarfism, severe growth plate disorganization, and intervertebral disc defects. In most animal models, global $\mathrm{KO}$ of the Arp $2 / 3$ complex or its activators causes early embryonic lethality (31). Arpc2 encodes the ARPC2/p34 subunit that is an essential component of all known Arp2/3 hybrid complexes (4). The ARPC2 subunit interacts with the preexisting "mother" filament and, thus, is required for nucleation and branch stability (32). In this study, we crossed the conditional Arpc2 allele, generated by flanking exon 8 of the Arpc2 gene with LoxP sites (33), to an established Col2-Cre driver (Col2-Cre; Arpc2 ${ }^{\mathrm{f} / \mathrm{fl}}$ ) (34) that targets intervertebral disc and cartilage as a way to delineate the physiological roles of Arp2/3 in these tissues (Figure 1A). Out of 89 pups with an expected Mendelian distribution of $1 / 4$, only 5 Col2-Cre; Arpc $2^{\mathrm{fl} / \mathrm{ll}}$ pups survived to genotyping performed at 7-10 days of age (Figure 1B). A mutant pup at P0 stained with $H \& E$ presented with decreased spacing of the ribcage and abnormal primary ossification centers in the sternum, suggesting that high embryonic lethality and low postnatal viability was caused by respiratory distress (Figure 1C). The surviving mutant mice showed severely stunted growth and presented with an abnormal curvature of the spine, seen clearly at P6 (Figure 1D) and P26 (Figure 1E). Safranin O staining of intervertebral disc sections from a P10 mutant showed either a strikingly small NP compartment or complete loss of NP, devoid of vacuolated notochordal cells with fused vertebrae (Figure 1F). Irrespective of the NP phenotype, the AF compartment was severely disorganized and deformed in all discs (Figure 1F). Strikingly, rounded chondrocyte-like cells were observed occupying the NP and inner AF regions (Figure 1F). Furthermore, Safranin O staining revealed marked expansion of vertebral growth plates characterized by disorganization of proliferative columns and loss of distinction between the cartilaginous end plate and growth plate (Figure $1 \mathrm{~F}$ ). We 
A

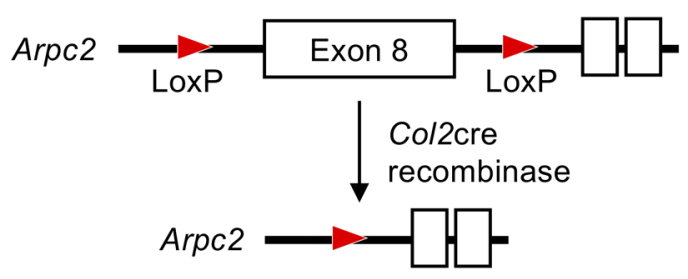

C

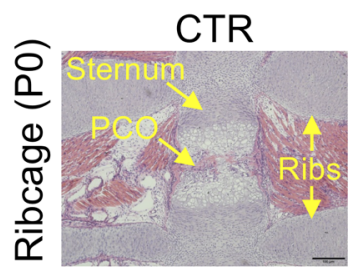

Col2-Cre-Arpc2 ${ }^{\mathrm{f} / \mathrm{f}}$

(cKO)

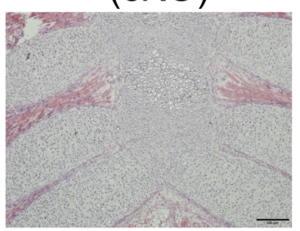

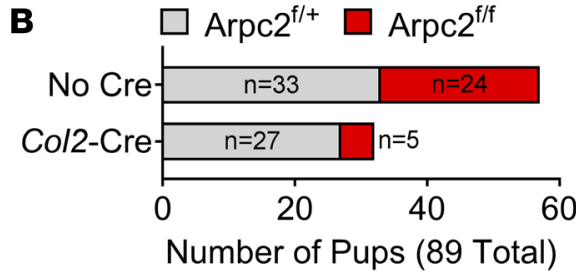
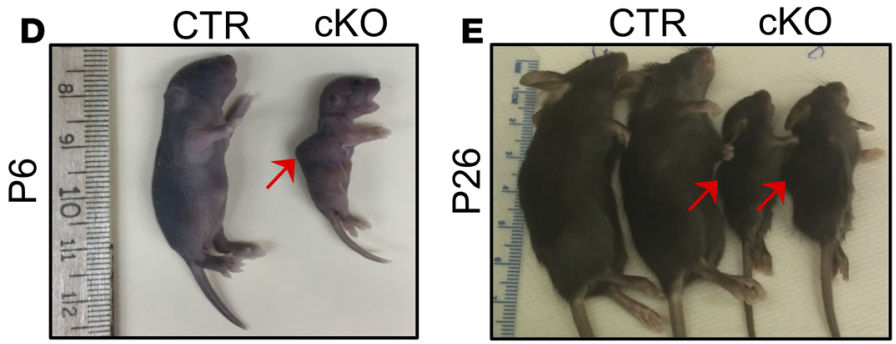

G $\quad$ CTR

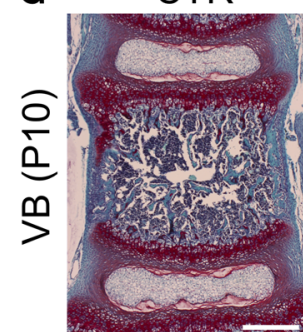

cKO

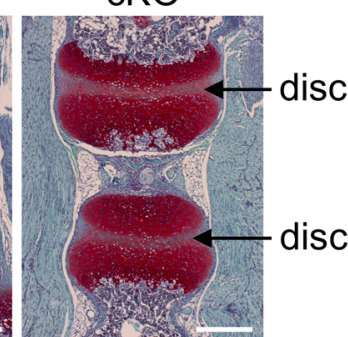

H
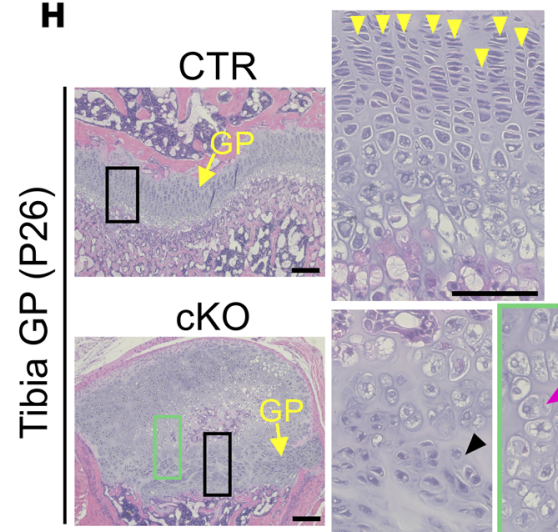
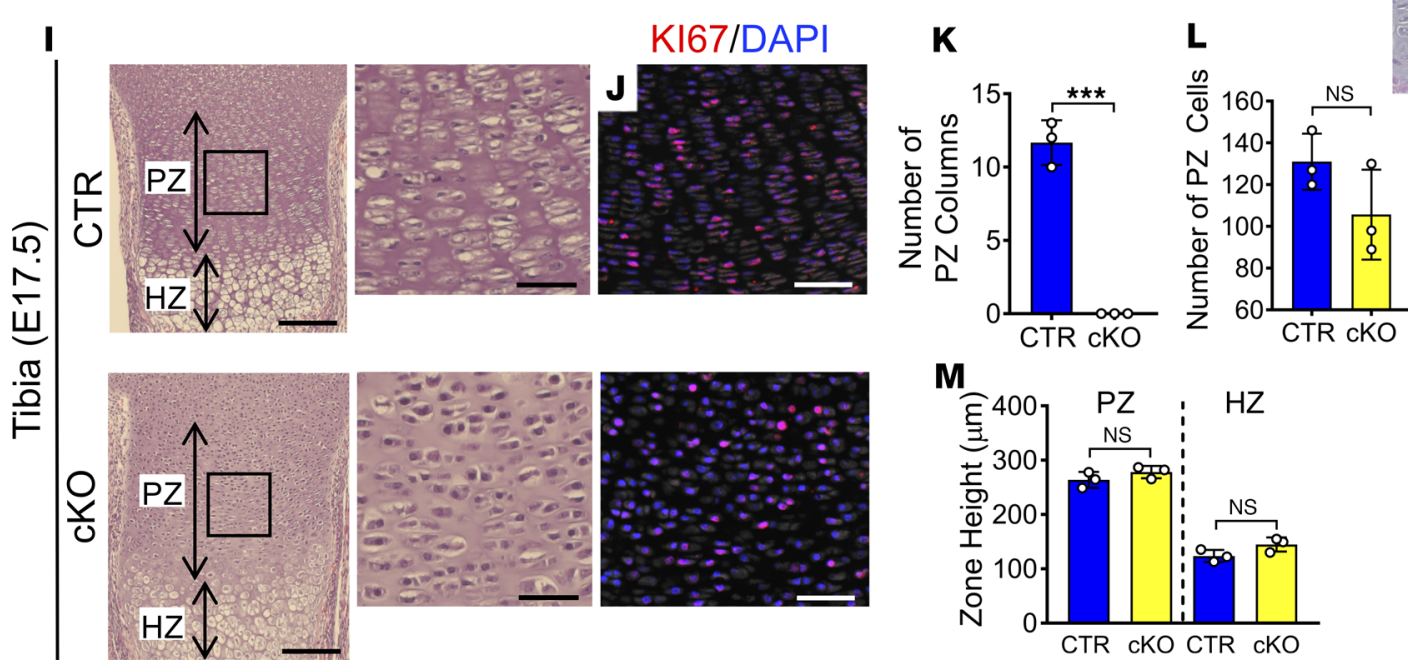
Figure 1. Constitutive inactivation of Arp2/3 in disc and cartilage causes severe defects. (A) Generation of Arpc2-null alleles in collagen II-expressing cells. (B) Frequency of pups that survived to genotyping with an expected Mendelian distribution of $1 / 4$ each. (C) H\&E-stained ribcage of mutant at PO compared with control (CTR). Scale bar: $100 \mu \mathrm{m} . n=1$. (D and E) Images of Arp2/3 mutant mice. P6, left panel; P26, right panel; shown next to littermate controls. Red arrows indicate abnormal curvature of the spine. (F) Coronal sections of discs from a P10 animal stained by Safranin 0/Fast Green/Hematoxylin. White outlines define the disc compartment. Boxes indicate higher-magnification images in bottom row, showing magnified end plates and growth plates. Yellow arrowheads show chondrocyte columns; white arrowheads show chondrocyte-like cells. Scale bars: $200 \mu \mathrm{m}$ in top row, $50 \mu \mathrm{m}$ in high-magnification view. $n$ $=1 ; 4$ discs. (C) Vertebrae at P10. Scale bar: $200 \mu \mathrm{m} . n=1 ; 6$ vertebrae. (H) H\&E-stained sections of tibia from P26 mouse ( $n=1)$. Yellow arrowheads indicate proliferative columns, black arrowheads indicate disorganization of cells, and pink arrowheads indicate chondrocytes with hypertrophic morphology. Scale bar: $100 \mu \mathrm{m}, 50 \mu \mathrm{m}$ in higher-magnification image. (I) H\&E-stained sections of embryonic tibia at E17.5. Scale bar: $100 \mu \mathrm{m}, 50 \mu \mathrm{m}$ in higher-magnification image. $n=3$ embryos. (J) Ki67 IHC on E17.5 tibia. Scale bar: $100 \mu \mathrm{m} . n=1$. (K) The number of proliferative columns containing 3 or more aligned cells were quantified from embryonic tibia. (L) In the same area, the number of cells in the proliferative zone were counted. (M) The heights of the proliferative zone (PZ) and hypertrophic zone (HZ) as shown in I were quantified. NP, nucleus pulposus; AF, annulus fibrosus; EP, end plate; GP, growth plate; VB, vertebral body. Quantitative measurements represent mean \pm SD. Significance was determined using unpaired Student's $t$ test. ${ }^{* * *} P \leq 0.01$.

also observed a marked decrease in the size of vertebral bodies (Figure 1G). Similar to the vertebrae, tibial growth plates showed severe growth plate disorganization with chondrocytes of abnormal morphology and a significant reduction in secondary ossification centers (Figure $1 \mathrm{H}$ ). Because chondrocytes at these early postnatal stages were clearly affected by Arpc2 loss, we assessed the consequence of deletion during embryonic development. In tibiae isolated from E17.5 embryos, the proliferative zone contained fewer organized columns without changes in overall cellularity and zone height, as well as significant disorganization of Ki67 ${ }^{+}$proliferative cells (Figure 1, I-M). These results suggest that Arp2/3 is critical for proliferative column organization during endochondral ossification.

To gain further insights into the disc and growth plate phenotype of Arp2/3-deficient mice, we investigated localization and abundance of collagen II, Sox9, and collagen X in P10 and P26 Col2-Cre; Arpc2 ${ }^{\mathrm{A} / \mathrm{l}}$ mutant mice. Collagen II staining in WT discs was robust in the cartilaginous end plate and inner AF, with little to no staining of the NP compartment (Figure 2A). On the other hand, mutant discs showed an absence of collagen II deposition in the end plate and robust staining of the disc core lacking distinction between NP and AF compartments, suggesting dysregulated matrix deposition (Figure 2A). In line with our observation that cells with a chondrocyte-like morphology occupied the $\mathrm{NP}$ and inner AF regions, these cells were positive for Sox9. Interestingly, however, while Sox 9 was expressed by most cells of the vertebral end plate and growth plate, the hypertrophic zone showed a substantial decrease in Sox 9 abundance (Figure 2B), with increased collagen $\mathrm{X}$ deposition at the growth plate-bone junction (Figure 2C). In tibia, Ki67 staining of the growth plate revealed a clear disorganization of proliferative chondrocytes (Figure 2D), similar to that observed in developing tibia at E17.5 (Figure $1 \mathrm{H}$ ). In addition, the regions of tibia lacking secondary ossification centers were occupied by Sox9 and collagen $\mathrm{X}^{+}$chondrocytes (Figure 2, E and F).

Given the extremely low survival of Col2-Cre; Arpc2 ${ }^{\mathrm{A} / \mathrm{fl}}$ mice, we next crossed the conditional Arpc2 allele to an inducible Cre driver that targets cartilage and disc to allow for investigation at postnatal time points. Acan-CreER ${ }^{\mathrm{T} 2}$ mice harboring a tamoxifen-inducible Cre recombinase driven by the aggrecan promoter (35) were crossed with $A r p c 2^{\mathrm{A} / \mathrm{fl}}$ (33) to generate Acan-CreER $\mathrm{R}^{\mathrm{T} 2}$; Arpc2 $2^{\mathrm{f} / \mathrm{fl}}$ mice. These mice were injected with tamoxifen at either P1 or P12 and evaluated at P19 (Figure 2G). H\&E staining of growth plates with Arp2/3 loss induced at P1 or P12 showed significant disorganization of the growth plate at P19 (Figure 2H), closely recapitulating the phenotype observed in Col2-Cre; Arpc2 ${ }^{\mathrm{A} / \mathrm{fl}}$ mice. The results also show that the growth plate phenotype of mice injected at $\mathrm{P} 1$ was more pronounced than mice injected at P12, suggesting that the phenotype severity was time dependent, wherein sufficient time was required for the Arpc2-null migrating cells to diverge from their columnar organization. Furthermore, growth plate chondrocytes were morphologically abnormal and significantly enlarged in mice injected with tamoxifen on day 1 , but they appeared morphologically normal when injected with tamoxifen on day 12 (Figure $2 \mathrm{H}$ ). Moreover, while Ki67 staining showed similar abundance to that observed in Col2-Cre; Arpc2 ${ }^{\mathrm{f} / \mathrm{fl}}$ mutant mice, Sox9 levels appeared lower and collagen X staining was nearly undetectable in the growth plate, indicating that postnatal loss of Arp2/3 driven by the aggrecan promoter had a slightly different effect on growth plate homeostasis than when Arp2/3 was constitutively lost in collagen II-expressing cells during early development (Figure 2, I-K).

Inactivationof Arp2/3atskeletalmaturityresultsinprematuregrowthplateclosureandarticularcartilagedefectsassociated with impaired chondrocytespreading. To evaluate the effect of Arp2/31oss at skeletal maturity, Acan-CreER ${ }^{\mathrm{T} 2}$; $\mathrm{Arpc}^{\mathrm{A} / \mathrm{fl}}$ mice were injected with tamoxifen at 4 months of age and evaluated at 6 or 12 months (Figure 3A). 
Intervertebral Disc (P10)
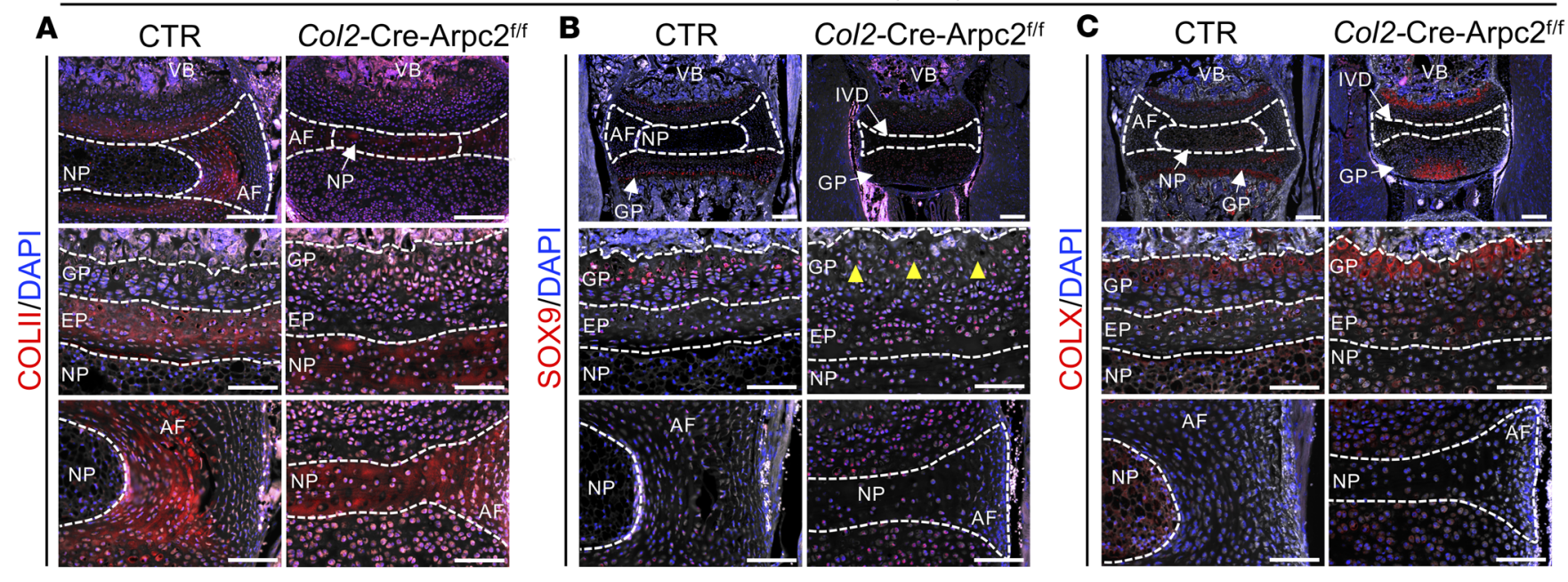

Tibia GP (P26)
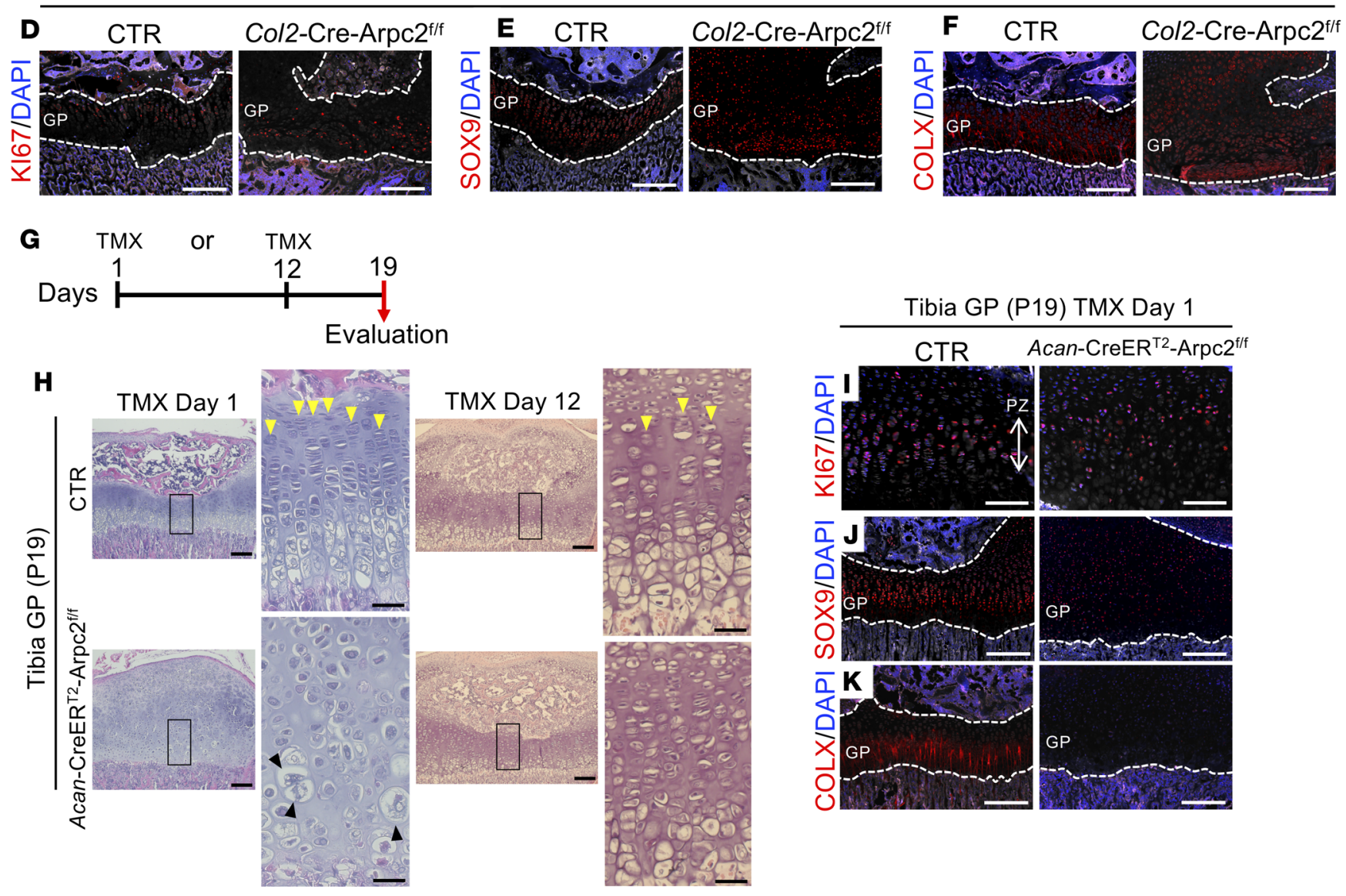

Figure 2. Impact of Arp2/3 inactivation on disc and chondrogenic cell markers. (A-C) IHC performed on P10 discs of Col2-Cre; Arpc2 $2^{\mathrm{fl} / \mathrm{fl}}$ mutant mice stained for collagen II (COLII) (A), Sox9 (B), and collagen X (COLX) (C). Scale bars: $200 \mu \mathrm{m}$ (top row); $100 \mu \mathrm{m}$ (middle and bottom rows). Yellow arrowheads indicate reduced Sox9 staining in the GP. $n=1 ; 4$ discs. (D-F) IHC staining of P26 tibia of Col2-Cre; Arpc2 ${ }^{\mathrm{fl} / \mathrm{fl}}$ mutant mice for Ki67 (D), Sox9 (E), and collagen X (F). Scale bar: $200 \mu \mathrm{m} . n=1$. (G) Timeline showing strategy for Arpc2 deletion using Acan-CreER ${ }^{\mathrm{T} 2}$, wherein pups were injected with tamoxifen (TMX) at P1 or P12 and evaluated at P19. (H) H\&E staining of tibial growth plate from P19 animals injected at either day 1 or day 12. Yellow arrowheads indicate proliferative columns. Black arrowheads indicate chondrocytes with hypertrophic morphology. Scale bar: $100 \mu \mathrm{m}$,

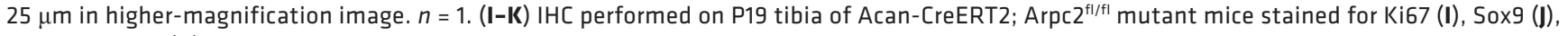
and collagen $X(\mathbf{K})$. Scale bar: $200 \mu \mathrm{m} . n=1$. 
A

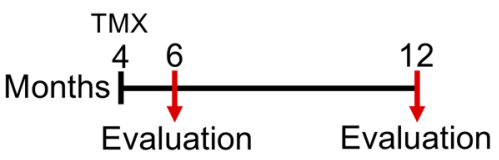

D
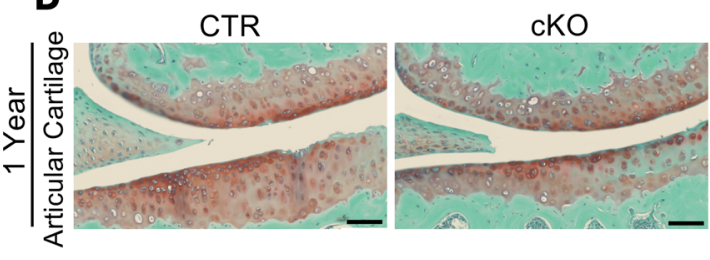

G Growth plate chondrocytes

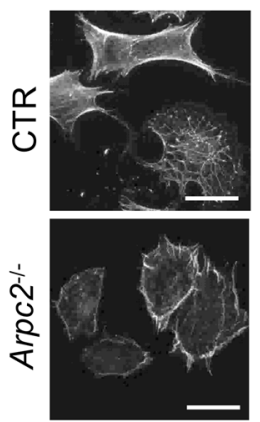

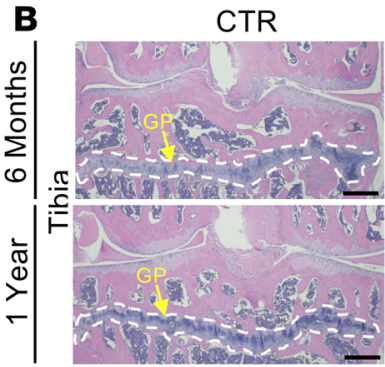

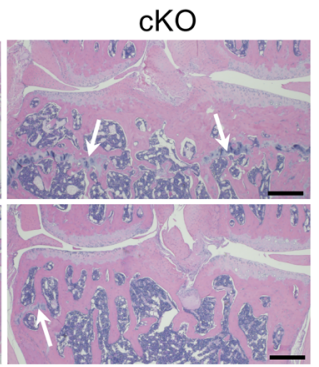

C
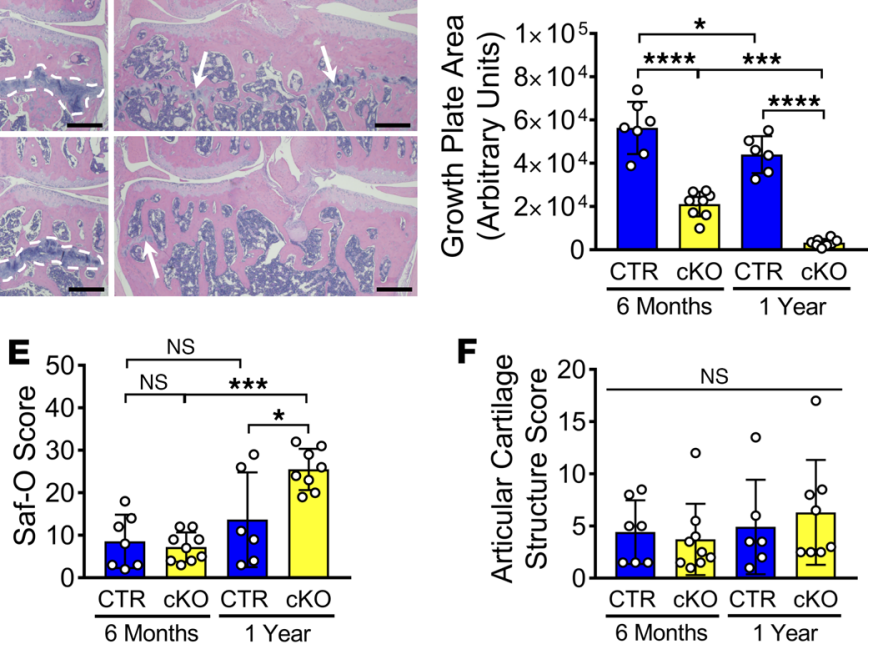

I Articular chondrocytes

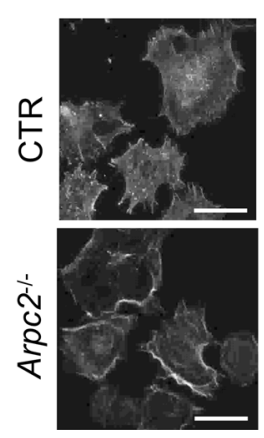

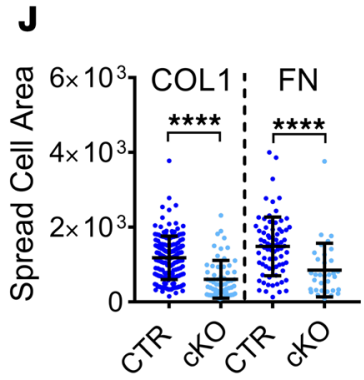

Figure 3. Postnatal inactivation of Arp2/3 causes growth plate closure and articular cartilage defects. (A) Acan-CreERT2 mice were injected with tamoxifen at 4 months and evaluated at 6 and 12 months of age. (B) H\&E-stained tibia sections of mice at 6 months and 1 year. White outline defines the growth plate (CP) in control animals; white arrows show reminiscent growth plate in mutant mice. Scale bar: $100 \mu \mathrm{m}$. $n \geq 6$. (C) Growth plate area was quantified by tracing in ImageJ. (D) Safranin 0/Fast Green staining of the articular cartilage in 1-year-old mice after tamoxifen-induced loss of Arp2/3 at 4 months as compared with controls. Scale bar: $50 \mu \mathrm{m} . n \geq 6$. (E and F) Stained slides were graded for proteoglycan loss (E) and articular cartilage structure (OARSI) (F). In both measures, scores are summed from each of 4 cartilage surfaces graded on a 12-point scale, where higher scores indicate worse OA. (G and I) High-magnification images of growth plate (C) and articular chondrocytes (I) isolated at P19 after induction of Arp2/3 loss at P12. Scale bar: $25 \mu \mathrm{m}$. (H and J) Spread cell area of growth plate $(\mathbf{H})$ and articular chondrocytes $(\mathrm{J})$ were quantified to determine the extent of interaction with the underlying collagen I (COL1) or fibronectin (FN). Quantitative measurements represent mean cell area \pm SD; $n \geq 33$ cells/group. Significance for growth plate areas and articular cartilage grades was determined by 1-way ANOVA and Kruskal-Wallis, respectively. Significance between cell spreading was determined using Mann-Whitney $U$ test; ${ }^{*} P \leq 0.05 ;{ }^{* * *} P \leq 0.001 ;{ }^{* * *} P \leq 0.0001$.

These mice showed moderate growth plate closure at 6 months and nearly complete loss of the growth plate by 1 year, as seen on histology (Figure 3B) and indicated by growth plate area quantification (Figure 3C). The hind limbs of 1-year-old mutant mice were also isolated and histologically scored to determine whether inactivation of Arp2/3 disrupted articular cartilage homeostasis (Figure 3D). Articular cartilage with $A r p c 2$ loss had a significant decrease in proteoglycan content when summed across the 4 quadrants of the joint: medial and lateral sides of the femoral and tibial surfaces (Figure 3E). There were no significant differences in the articular cartilage structure score (Figure 3F; ref. 36), and osteophytosis was not noted in either group, suggesting that overall joint integrity was maintained. To gain mechanistic insight into the role of Arp2/3 in chondrocyte function, we used flow cytometry to sort growth plate and articular chondrocytes from P19 Acan-CreER ${ }^{\mathrm{T} 2}$; Arpc2 $2^{\mathrm{f} / \mathrm{fl}}$ mice that had been injected with tamoxifen at P12. To ensure that the cells investigated had loss of $A r p c 2$, we included a ROSA-lox-stop-lox ZsGreen Cre reporter allele in the crosses and sorted ZsGreen ${ }^{+}$cells. Growth plate and articular chondrocytes were separately plated on either collagen I- or fibronectin-coated substrates. Unlike WT cells that showed distinct lamellipodia, Arpc2-null cells showed diffuse and less organized actin structures and an overall rounded morphology, 
without conspicuous changes in overall actin content (Figure 3, G and I). Arpc2-null growth plate and articular chondrocytes showed a marked reduction in spreading, irrespective of the substrata (Figure $3, \mathrm{H}$ and J), suggesting that Arp2/3 function was essential for normal interactions with ECM or was required for the transduction of signals elicited by substrate binding. To further determine whether the inducible loss of Arpc2 in chondrocytes of skeletally mature mice affected the overall abundance of actin in vivo, we used confocal microscopy $\mathrm{Z}$ stacks to assess phalloidin staining in cartilage of femoral condyles harboring cells with and without successful recombination by the Cre driver. Two months after the injection of a low dose of tamoxifen, approximately half of the cells had Arpc2 loss, as indicated by the ZsGreen signal resulting from recombination of the ROSA-lox-stop-lox ZsGreen allele. Quantification of actin levels were not different between cells that had successfully recombined (Arpc2 loss) and those that had not recombined (Arpc2 intact) (Supplemental Figure 1, A and B; supplemental material available online with this article; https:// doi.org/10.1172/jci.insight.131382DS1).

Postnatal inactivation of Arp2/3 driven by Acan-CreER ${ }^{T 2}$ causes intervertebral disc degeneration. To assess the function of Arp2/3 in intervertebral discs of skeletally mature animals, Acan-CreER ${ }^{\mathrm{T} 2}$; Arpc2 $2^{\mathrm{f} / \mathrm{fl}}$ mice were injected with tamoxifen at 4 months of age and evaluated at 6 or 12 months. Histological analyses of 6-month-old mutant discs showed a modest effect on the NP compartment, evident by the presence of vacuolated notochordal NP cells in the majority of discs, and bulging proteoglycan-rich matrix (Figure 4, $\mathrm{A}$ and B). There was also a moderate loss of demarcation between the NP and inner AF compartments, and there was partial growth plate closure in both lumbar (Figure 4, A and B) and caudal motion segments in 6-month-old mutant mice (Supplemental Figure 2). Interestingly, 24\% of 6-month-old lumbar discs in mutant mice presented with abnormally enlarged NP cells (Figure 4C). By 1 year of age, despite containing relatively larger cells, the lumbar NP showed a typical morphology (Figure 4, D and E). Furthermore, NP cell phenotypic markers carbonic anhydrase 3, keratin-19, and vimentin were not affected by Arp2/3 deletion (Supplemental Figure 3). However, 1-year-old lumbar discs showed further loss of $\mathrm{NP} / \mathrm{AF}$ demarcation, deterioration of AF lamellae, and nearly complete loss of vertebral growth plates (Figure 4E). Consistent with AF degeneration, a few mice lacking Arp2/3 showed spontaneous annular tears, which were absent in control animals (Figure $4 \mathrm{~F}$ ). Interestingly, caudal discs of 1-year-old mutant animals showed a high degree of degeneration, characterized by marked fibrosis and inward buckling of the AF lamellae (Figure 4, G and H). Histological changes in Arp2/3-deficient discs were scored using the modified Thompson scale as previously described (37, 38). Average grades (Figure 4 , I and J) and grade distributions (Figure 4, $\mathrm{K}$ and L) clearly showed increased AF grades in lumbar and caudal discs at both time points, whereas NP grades only showed a trend of higher scores in 1-year-old caudal discs. The intrinsic intra- and interanimal variation in disc degeneration phenotypes of lumbar and caudal discs at 6 month and 1 year was noted and is shown in Supplemental Figure 2. Indeed, genetic interactions with environmental stimuli have been indicated to substantially differ between lumbar levels in twin studies, and the biomechanical environment has been shown to differ significantly level by level along the spinal column, resulting in level-specific phenotypic outcomes $(30,39-42)$. Although it is plausible that the phenotypic variability between animals was caused by some variation in recombination efficiencies of the floxed allele, the overall recombination is high, regardless of the spinal level. Consistent with our histological observations, the area of the NP cell band (Figure 4M) and the number of NP cells (Figure 4N) were increased in 1-year-old Arpc2-null animals. To assess cell death as a consequence of Arpc2 deletion, we performed TUNEL staining on 1-year-old lumbar discs; these stainings showed only a few TUNEL ${ }^{+}$cells in the NP and AF compartments of both control and mutant animals (Figure 4O). Additionally, to delineate the fate of disc and vertebral growth plate cells in mutant mice, we performed lineage tracing studies using a ROSAlox-stop-lox ZsGreen Cre reporter. At 6 months, ZsGreen signal was robust in nearly all cells of the NP, AF, and growth plate, suggesting that these were the same cells that were targeted at 4 months or descendant cells from those that were targeted (Supplemental Figure 4). Interestingly, at 1 year, ZsGreen ${ }^{+}$cells were either fully or partially retained in NP and AF tissues, depending on the degree of degeneration, and were markedly lost in the growth plate (Figure 4, P and Q). However, we did not observe repopulation of the disc tissues with nonlabeled cells in mutant mice, even with severe degeneration following Arpc2 deletion (Figure 4, P and Q). The earlier-described cell spreading assay showed that cell-ECM interactions were compromised in Arpc2-null chondrocytes; therefore, we hypothesized that cell-ECM interactions were also compromised in disc cells. Interestingly, NP cells showed a robust decrease in cell spreading on collagen 1 but not fibronectin substrate when treated with Arp2/3 inhibitor (Figure 4, R and S). These data suggest 


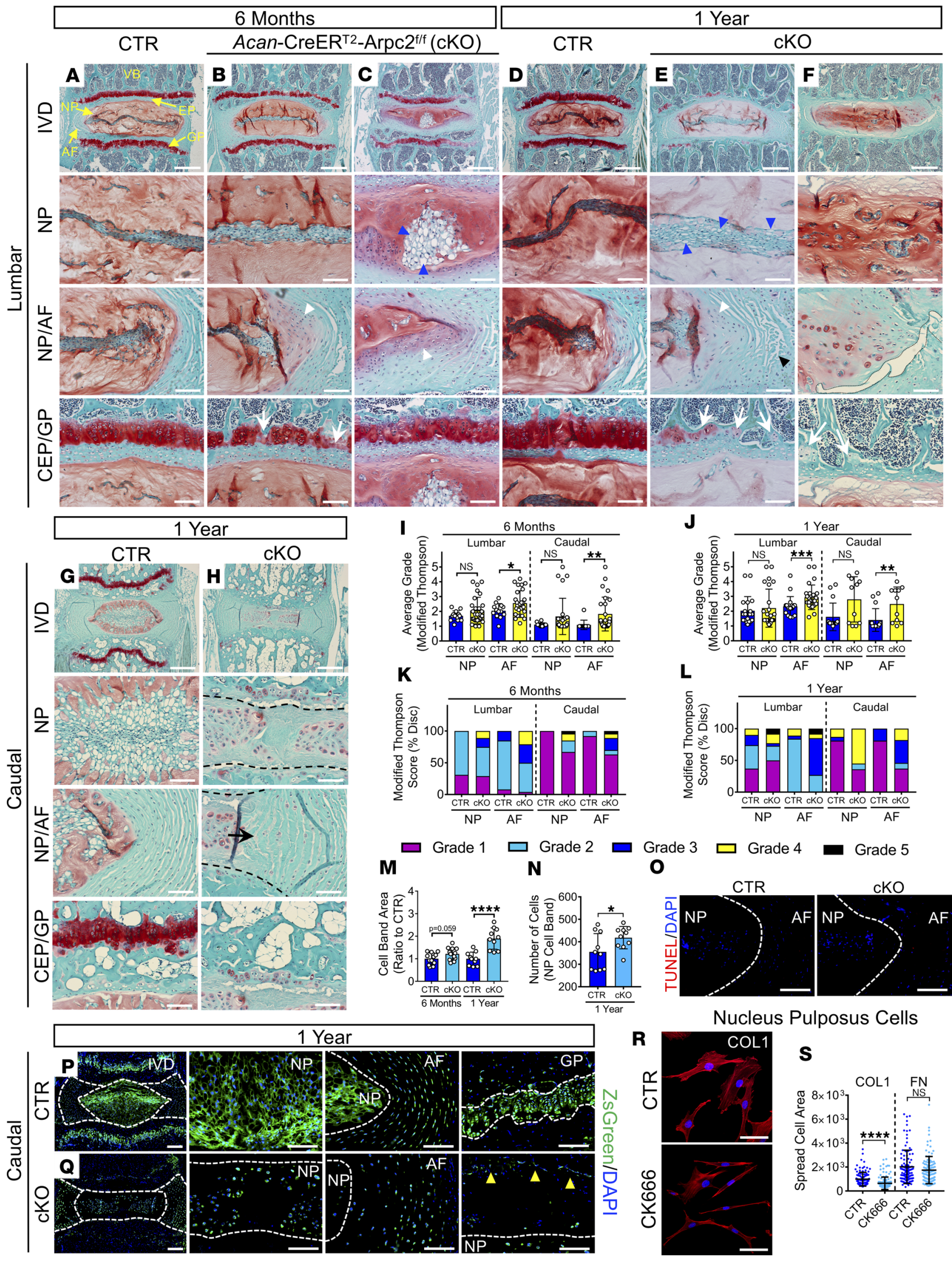


Figure 4. Inactivation of Arp2/3 at skeletal maturity causes disc degeneration. (A-F) Coronal sections of control and Arp2/3-deficient discs stained by Safranin 0/Fast Green/Hematoxylin. Scale bar: $200 \mu \mathrm{m}$ in top row, $100 \mu \mathrm{m}$ in high magnification. (A-C) Lumber discs from 6-month-old control (A) and mutant mice (B and $\mathbf{C}$ ). White arrowheads indicate loss of demarcation between the NP and AF; white arrows indicate moderate loss of the growth plate ( $n=7$ mice). Blue arrowheads indicate enlarged NP cells. (C) A total of $24 \%$ of 6 -month-old mutant lumbar discs presented with abnormally enlarged NP cells (blue arrowheads). (D-F) Lumbar discs from 1-year-old control (D) and mutant discs (E and F). Blue arrowheads indicate enlarged NP cells; white arrowhead indicates loss of demarcation between the NP and AF compartments; black arrowhead indicates disorganization of AF lamellae; white arrows indicate reminiscent growth plate $(n=6$ mice). (F) Annular tear (outlined in black). (C) One-year-old control for caudal discs. (H) Severe disc degeneration with condensed fibrotic matrix in 1-year-old mutant discs ( $n=3$ mice). Severely degenerated caudal discs showed inner buckling of the AF (black arrow). (I and J) Average modified Thompson score for 6-month-old ( $n=28$ lumbar and 27 caudal discs; 7 mice) (I) and 1-year-old animals ( $n=24$ lumbar discs; 6 mice and $n=11$ caudal discs; 3 mice) (J), where higher scores indicate worsening changes. (K and $\mathbf{L}$ ) Distribution of histological grades 6-month-old (K) and 1-year-old (L) animals. (M) Area measurements of the NP cell band of lumbar discs ( 6 months, $n=12$ discs, 3 mice; 1 year, $n=10$ discs, 5 mice). (N) Cell number in the NP cell band ( $n=10$ discs; 5 mice). (0) TUNEL assay of 1-year-old lumber discs. Scale bar: $100 \mu \mathrm{m}$. (P and $\mathbf{Q})$ Fate mapping using ZsGreen in 1-year-old control (P) and mutant (Q) caudal discs. Scale bar: $200 \mu \mathrm{m}$ in left column, $100 \mu \mathrm{m}$ in high-magnification view, to the right. Yellow arrowheads indicate GP loss. (R and $\mathbf{S}$ ) Spread cell area of NP cells was quantified to determine the extent of interaction with underlying collagen I (COL1) or fibronectin (FN). Scale bar: $50 \mu \mathrm{m}$. Quantitative measurements represent mean \pm SD. Significance was determined using either unpaired Student's $t$ test or Mann-Whitney $U$ test; ${ }^{*} P \leq 0.05 ;{ }^{* *} P \leq 0.01 ;{ }^{* *} P \leq 0.001 ;{ }^{* * *} P \leq 0.0001$.

that compromised cell-ECM interactions or transduction signals elicited by matrix adhesion may have contributed to the disc degeneration phenotype of Acan-CreER ${ }^{\mathrm{T} 2}$; Arpc2 ${ }^{\mathrm{f} / \mathrm{fl}}$ mice, and they highlight the cell type-specific differences between chondrocytes and NP cells in their interaction with matrix constituents.

Disc degeneration in Arp2/3-deficient mice is characterized by aberrant abundance and deposition of ECM proteins in the annulus fibrosus. Because the AF compartment of Acan-CreER ${ }^{\mathrm{T} 2}$; Arpc2 $2^{\mathrm{t} / \mathrm{fl}}$ mice showed clear evidence of degeneration, we further delineated changes in ECM composition of the disc. Total collagen content and collagen fiber orientation were visualized by Picrosirius red staining using bright-field (Figure $5 \mathrm{~A}$ ) and polarized light microscopy (Figure 5B). The AF of mutant mice showed disorganized lamellar architecture without overt changes in the distribution of collagen fiber thickness (Figure 5, B and C). Moreover, quantitative IHC revealed no changes in collagen I and II (Figure 5, D, E, K, and L). Interestingly, however, the mutant mice showed increased staining of a fluorescently labeled collagen hybridization peptide that specifically binds to denatured collagen (Figure 5, F and M), as well as decreased levels of cartilage oligomeric matrix protein (COMP), a key player in maintaining the organization of collagen fibers and the structural integrity of cartilaginous tissues (Figure 5, G and N and ref. 43). Furthermore, our analysis of the AF showed moderately decreased levels of collagen IX, a collagen required for disc maintenance (Figure 5, $\mathrm{H}$ and $\mathrm{O}$ ), as well as increased collagen X (Figure 5, I and P), a marker associated with disc aging and degeneration (44). Arp2/3-deficient discs also showed increased AF staining of ARGxx, a neoepitope that is exposed following aggrecanase-dependent degradation of aggrecan core protein (Figure 5, J and Q). In contrast to changes seen in the AF, NP tissue of 1-year-old mutant mice showed comparable levels of aggrecan, chondroitin sulfate, collagen X, and collagen IX to controls (Supplemental Figure 5).

Arp $2 / 3$ and Cdc42 control the activity of osmoregulatory transcription factor TonEBP. Since previous reports have implicated Arp2/3 and its upstream Rho-GTPases in the osmotic stress response (5, 21, 45-47), we hypothesized that activity of TonEBP, the only known osmoregulatory transcription factor in mammals, is regulated by Arp2/3. Immunofluorescence of 1-year-old Acan-CreER ${ }^{\mathrm{T} 2} ; \operatorname{Arpc}^{\mathrm{A} / \mathrm{fl}}$ discs showed a downward trend in TonEBP protein abundance without reaching significance in NP cells (Figure 6, A and B). However, TauT, a well-established osmosensitive target of TonEBP, showed decreased levels in Arp2/3-deficient discs compared with controls (Figure 6, C and D), suggesting that Arp2/3 plays a role in the regulation of TonEBP activity. Western blot and subsequent densitometric analysis also revealed that the hyperosmotic increase in TonEBP protein levels was reduced by inhibition of Arp2/3 with CK666 (Figure 6, E and F; see complete unedited blots in the supplemental material). To gain further mechanistic insights into the regulation of TonEBP by Arp2/3, we performed in vitro loss-of-function studies using primary NP cells, which make up an ideal model to study osmoadaption as physiological osmolarity is markedly elevated in the NP compartment $(24,48,49)$. Particularly, we investigated whether inhibition of Arp2/3 (CK666), Cdc42 (ML-141), or Rac1-GEF (NSC23766) by specific inhibitors control TonEBP transactivation domain (TAD) activity, a measure of its ability to recruit transcriptional cofactors. TonTAD activity was measured using a binary GAL4 system illustrated in Figure 6G. Treatment with Arp2/3 or Cdc42 inhibitor showed a significant reduction in NaCl-induced TonTAD activity; however, Rac1-GEF inhibition had no effect (Figure $6 \mathrm{H}$ ). This result provides strong evidence that TonEBP transactivational activity is regulated by Arp $2 / 3$ and Cdc42 under hyperosmolar conditions. We then measured the promotor activity of 3 well-described 
A
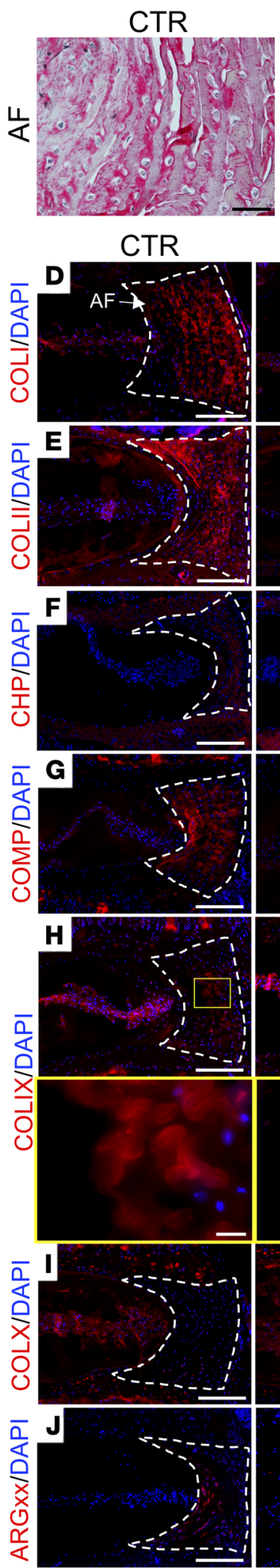
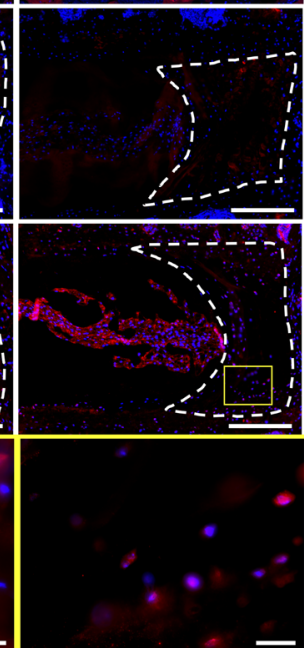

B AcanCreER ${ }^{\mathrm{T}} ; \mathrm{p} 34^{\mathrm{f} / \mathrm{f}}(\mathrm{cKO})$

$\mathrm{cKO}$
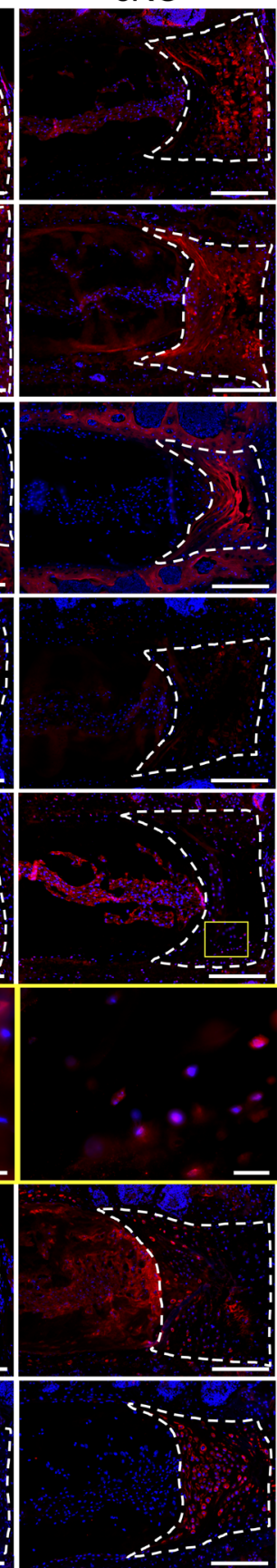

K

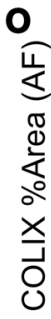

\section{Q}

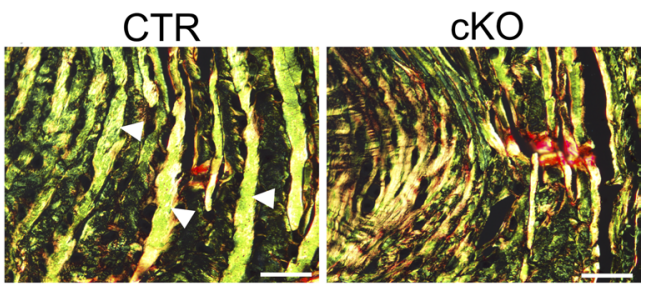

C

C

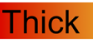

CTR

$\mathrm{cKO}$
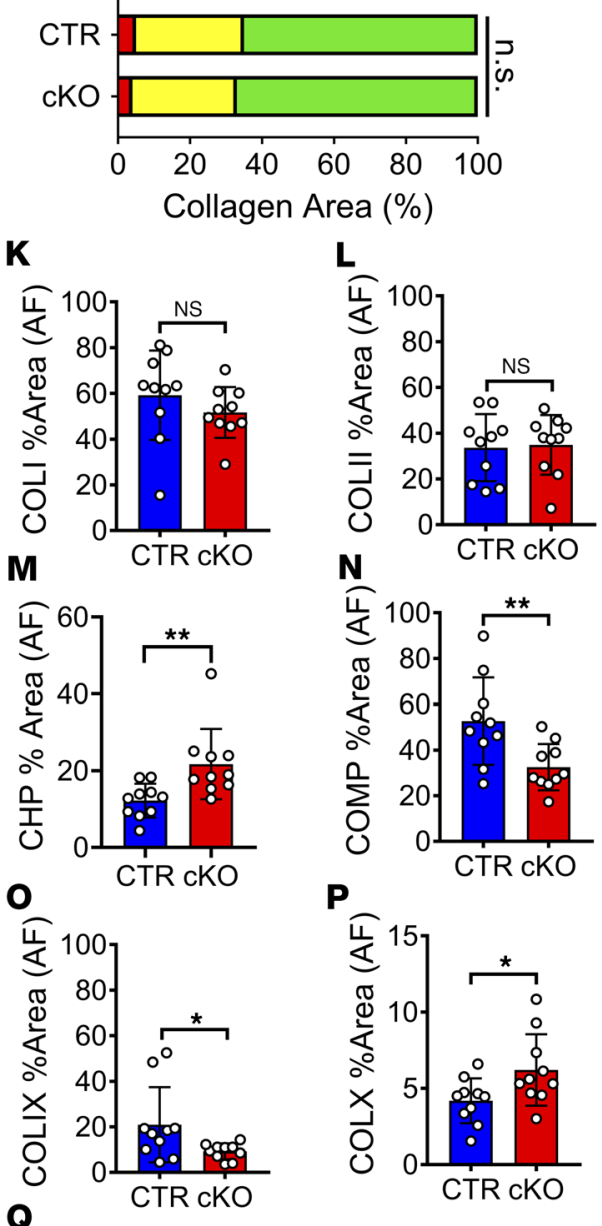

N

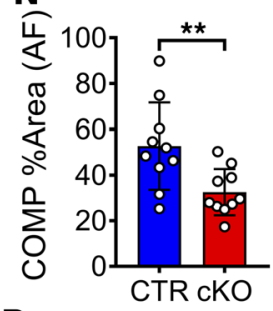

P

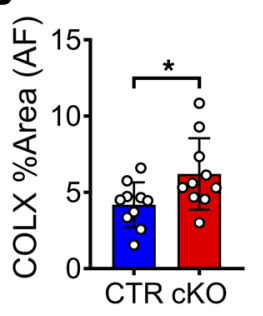

Figure 5. Annulus fibrosus degeneration in Arp2/3-deficient mice is characterized by altered extracellular matrix content. (A) Picrosirius red staining of 1-year-old lumbar discs showing collagen content in the AF. Scale bar: $50 \mu \mathrm{m}$. $n$ $=10$ discs; 5 mice. (B) Collagen fibers visualized under polarized light. Scale bar: $50 \mu \mathrm{m}$. White arrowheads indicate lamellae. (C) Quantification of collagen fiber thickness distribution.

(D, E, K, L) Quantitative IHC performed on 1-year-old lumbar discs stained by collagen I (COLI) ( $\mathbf{D}$ and $\mathbf{K}$ ) and collagen II (COLII) ( $\mathbf{E}$ and $\mathbf{L}$ ). ( $\mathbf{F}$ and $\mathbf{M}$ ) Collagen degradation analyzed by staining with fluorescently conjugated collagen hybridization peptide (CPH). (G-J and N-Q) Quantitative IHC performed on 1-year-old lumbar discs stained by cartilage oligomeric matrix protein (COMP) ( $\mathbf{G}$ and $\mathbf{N}$ ), collagen IX (COLIX) ( $\mathbf{H}$ and $\mathbf{O}$ ), collagen $X(C O L X)$ (I and $\mathbf{P}$ ), and ARGXx (J and $\mathbf{Q}$ ). Scale bar: $100 \mu \mathrm{m} . ~ n=10$ discs; 5 mice. Quantitative measurements represent mean \pm SD. Significance was determined using either unpaired Student's $t$ test or Mann-Whitney $U$ test. $\chi^{2}$ test was applied where graph represents contingency plot. ${ }^{*} P \leq$ $0.05 ;{ }^{* *} P \leq 0.01$

TonEBP osmosensitive target genes in the disc: TauT, Hsp70, and AR. These promoters contain highly conserved and well-characterized TonEBP binding elements (TonE). Treatment with Arp2/3 and Cdc42 inhibitors decreased the activity of all 3 reporters (Figure 6, I-K). Next, to determine the specificity of the response, we transfected NP cells with TauT or Hsp70 reporter plasmids harboring mutations within 

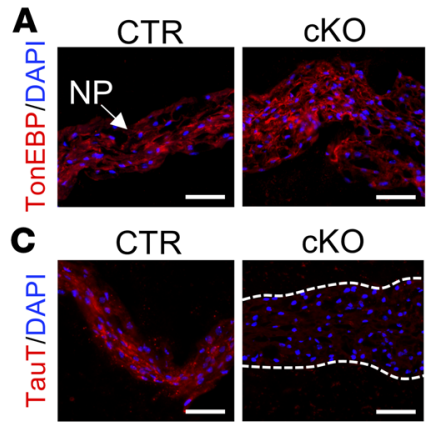

cKO

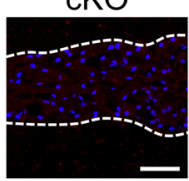

B

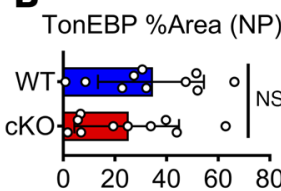

D

TauT \%Area (NP)

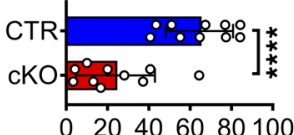

$\mathbf{E}$

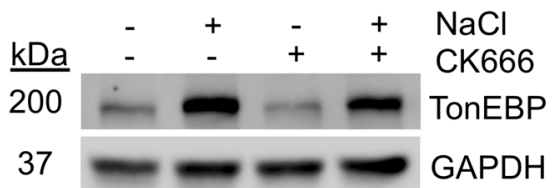

$\mathbf{F}$

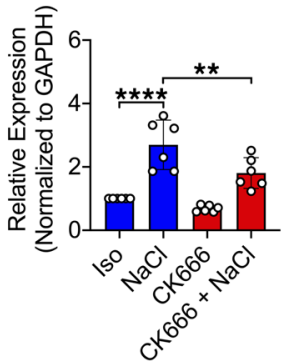

G
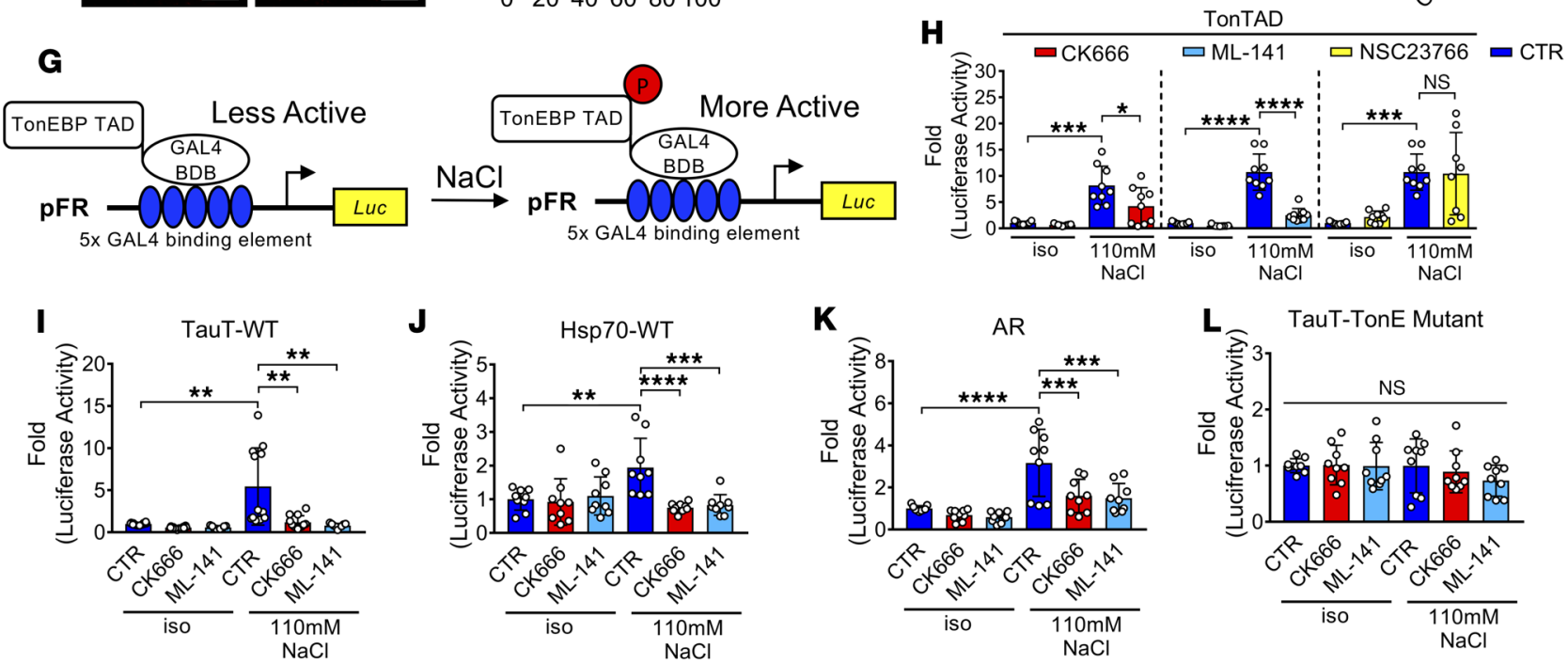

$\mathbf{L}_{\widehat{A}}$ TauT-TonE Mutant
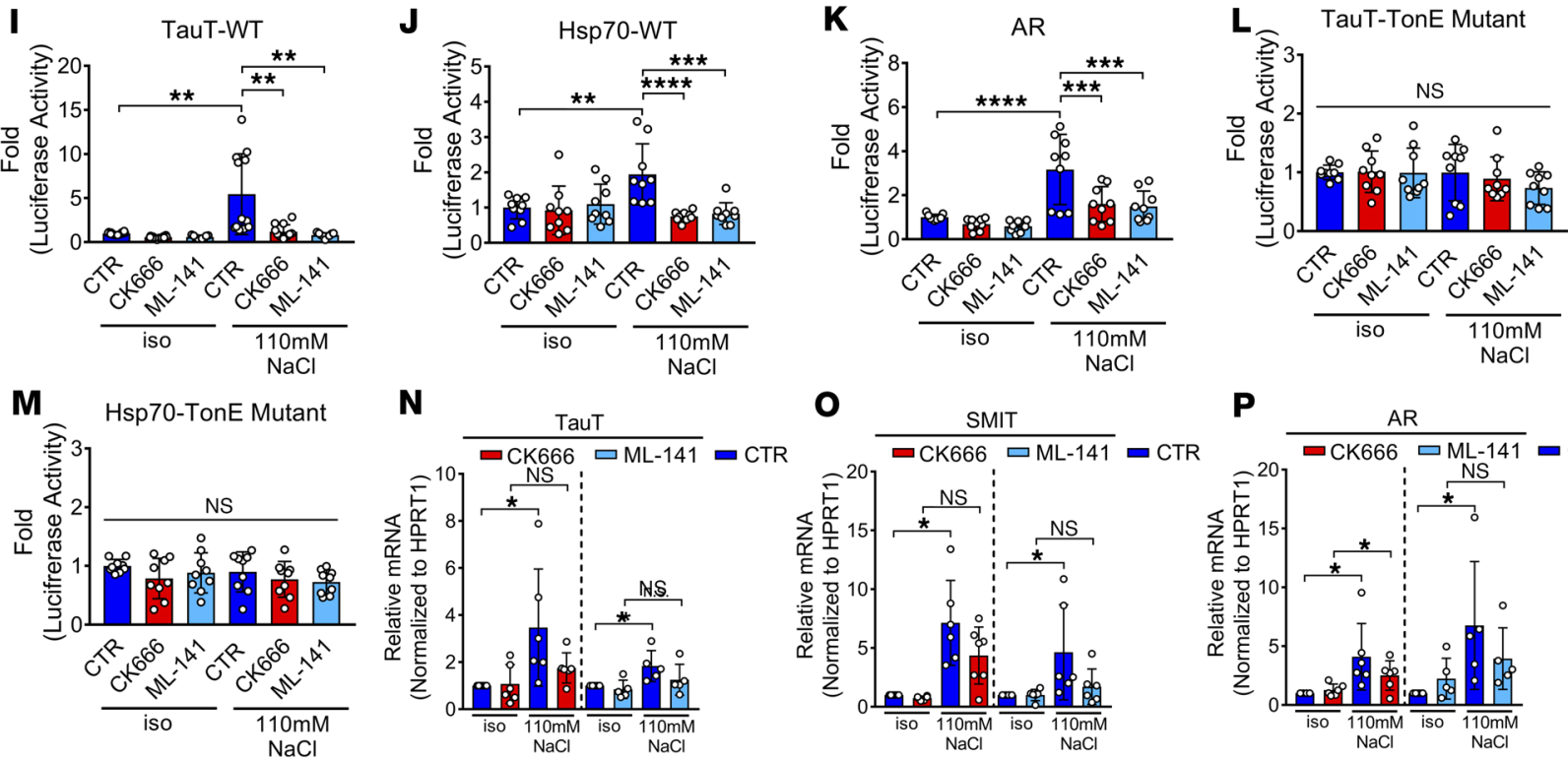

$\mathbf{N}$
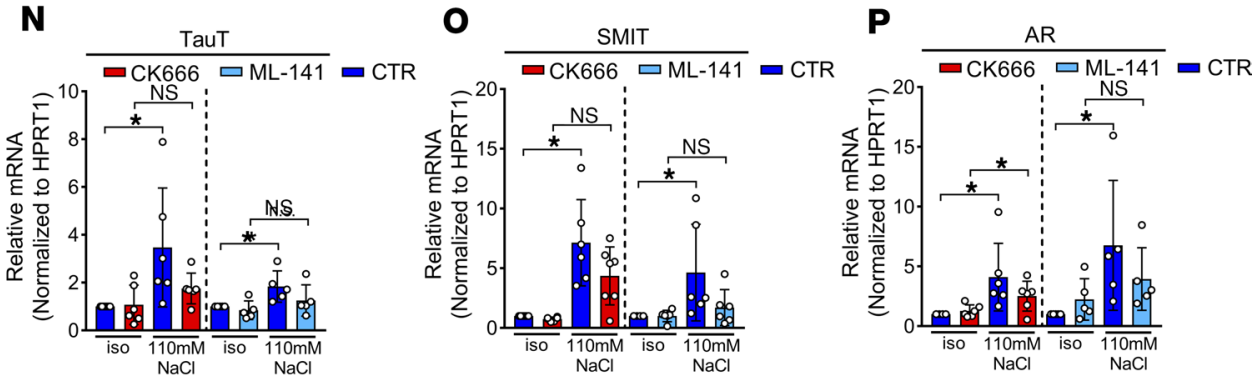

Figure 6. Arp2/3 and Cdc42 control TonEBP activity. (A-D) Quantitative immunohistostaining of TonEBP ( $\mathbf{A}$ and $\mathbf{B}$ ) and TauT ( $\mathbf{C}$ and $\mathbf{D}$ ) in NP cells of 1-year-old lumbar discs. Scale bar: $100 \mu \mathrm{m}$. $n=10$ discs; 5 mice. (E and F) Western blot and densitometric analysis showing reduced TonEBP induction with hypertonic stimulation ( $n=6$ independent experiments). (C) Depiction of the binary TonTAD-GAL4 system used to measure TonTAD activity. (H) NaCl-induced activation of TonTAD with inhibition of Arp2/3 (CK666), Cdc42 (ML-141), and Rac1-CEF (NSC23766) in primary NP cells. (I-K) Luciferase assays measuring TauT (I), Hsp70 (J), and AR (K) promoter activities with $\mathrm{NaCl}$ treatment and $\mathrm{Arp} 2 / 3$ or $\mathrm{Cdc} 42$ inhibition ( $n=3,3$ replicates/experiment). ( $\mathbf{L}$ and $\mathbf{M}$ ) Luciferase assay measuring TauT (L) and Hsp70 (M) mutant promoter activity with $\mathrm{NaCl}$ treatment and Arp2/3 or Cdc42 inhibition ( $n=3,3$ replicates/experiment). ( $\mathbf{N}-\mathbf{P})$ Quantitative PCR measuring mRNA levels of TauT (N), SMIT (0), and AR (P) from primary NP cells treated with $\mathrm{NaCl}$ and Arp2/3 or Cdc42 inhibitors ( $n \geq 5$ independent experiments). (Q) Mechanistic model depicting Arp2/3-mediated regulation of TonEBP. Quantitative measurements represent mean \pm SD. Significance was determined using 1-way ANOVA or Kruskal-Wallis, when making multiple comparisons between groups, or unpaired Student's $t$ test, when making comparisons between only 2 groups. ${ }^{*} P \leq 0.05 ;{ }^{* *} P \leq 0.01$; ${ }^{* *} P \leq 0.001$; ${ }^{* * * *} P \leq 0.0001$. 
the TonE sites. Both $\mathrm{NaCl}$ and inhibitors did not affect the activity of these mutant reporters (Figure 6, $\mathrm{L}$ and $\mathrm{M}$ ). Furthermore, the hyperosmolarity-dependent increases in TauT and SMIT mRNA levels were diminished by inhibition of Arp2/3 and Cdc42 (Figure 6, N and O). Although Cdc42 inhibition blocked the NaCl-dependent increase in AR mRNA levels, treatment with an Arp2/3 inhibitor showed only a downward trend (Figure 6P). Furthermore, we observed that NaCl-induced TonEBP nuclear translocation was not impaired by Arp2/3 inhibition, suggesting that regulation of TonEBP activity in this context was independent of its nuclear translocation (Supplemental Figure 6). Next, because it has been previously shown that Rac1 mediates TonEBP activity under hyperosmotic conditions via phospholipase C- 1 1 (PLC- §1) (45), we treated NP cells with PLC-y1 inhibitor (U73122). The results show that PLC inhibition failed to block hyperosmotic induction of TauT-WT, Hsp70-WT, and AR luciferase reporters at a lower inhibitor concentration, with a small inhibitory effect only on TauT-WT and Hsp70-WT activity at higher concentration (Supplemental Figure 7, A-C). Interestingly, however, PLC inhibition did not result in any changes in mRNA levels of TauT, SMIT, and AR under hypertonic conditions at both concentrations of the inhibitor (Supplemental Figure 7, D-F). These results are consistent with our observation that the Rac1-GEF axis did not affect TonEBP activity in NP cells.

Phosphorylation of p38 and ERK1/2 has been shown to increase upon hypertonic stimulation of NP cells, and Arp2/3-depleted and CK666-treated cells have been shown to have sustained activation of p38 under isotonic conditions $(5,26)$. Therefore, since the activity of TonEBP is also responsive to p38 and ERK1/2 pathways, which are implicated in Cdc42 signaling, we assessed how these pathways responded to Arp2/3 inhibition in NP cells $(26,50)$. Interestingly, while phosphorylation of p38 was increased with $\mathrm{NaCl}$ treatment, the observed induction was significantly blocked by cotreatment with CK666, suggesting that Arp2/3 is required for hypertonicity-induced p38 activation in NP cells (Supplemental Figure 8, A and C). In contrast, treatment with $\mathrm{CK} 666$ with or without $\mathrm{NaCl}$ prolonged phosphorylation of ERK in comparison with induction by $\mathrm{NaCl}$ alone (Supplemental Figure 8, $\mathrm{B}$ and $\mathrm{D}$ ). Taken together, we propose a mechanistic model in which Cdc42 modulates TonEBP activity through Arp2/3 and p38, independent of the Rac1 and PLC pathway, demonstrating a potentially novel function for Arp $2 / 3$ in controlling the osmoadaptive response in NP cells (Figure 6Q).

\section{Discussion}

In this study, we report that conditional targeting of the actin regulator Arp2/3 in cartilage and the intervertebral disc results in severe growth plate defects, loss of proteoglycan content in articular cartilage, and complex changes to the NP and annulus fibrosus. These results underscore the central role of Arp2/3 in the homeostasis of cartilaginous tissues. Mechanistically, we show that Arp2/3 controls the activity of the osmosadaptive transcription factor, TonEBP, furthering our understanding of the contributions of Arp $2 / 3$ in osmoadaptation.

The phenotype of Arp2/3 loss was most pronounced in Col2-Cre; Arpc $2^{\mathrm{f} / \mathrm{fl}}$ mice. Their dwarfism seemed to arise from an inability of chondrocytes to flatten into their characteristic discoid shape and arrange into proliferative columns in the growth plate, consequently affecting endochondral ossification. The failure of column organization was likely caused by impaired cell-ECM interactions, as isolated Arpc2-null growth plate and articular chondrocytes showed reduced spreading on collagen and fibronectin substrata, both of which are important ligands for integrins on chondrocytes. The growth plate disorganization with Arpc2 loss is similar to the phenotypes seen with cartilage-specific inactivation of other critical mediators of cell-ECM interactions, such as Rac1, Cdc42, and $\beta 1$ integrin $(10,12,51)$. Arp2/3 may link integrin engagement to actin polymerization through the recruitment of Arp2/3 to vinculin, which can be mediated by Rac1 and PI3K (19, 20). In addition to growth plate disorganization during development, impaired cell-ECM interactions could explain the premature growth plate closure in 6-month-old and 1-year-old Acan-CreER ${ }^{\mathrm{T} 2} ; \mathrm{Arpc} 2^{\mathrm{f} / \mathrm{l}}$ mice. In the vertebrae and tibia, disorganization of the growth plate also appeared to be caused by an accelerated progression from the proliferative to hypertrophic stages of chondrocyte differentiation, as the growth plates and chondrocytes that occupied the secondary ossification field contained an overabundance of collagen X-expressing cells. Since Sox 9 has been shown to inhibit proliferative chondrocytes from entering the hypertrophic stage (52), this acceleration might be explained by the lack of Sox9 levels in Arp2/3-deficient chondrocytes, as observed in the vertebral growth plate. Furthermore, this explanation is consistent with previous work that has shown that loss of $\beta 1$ integrin in early limb mesenchyme causes terminal differentiation of chondrocytes with associated actin cytoskeleton disruption (17). In articular cartilage, Arp2/3 inactivation resulted in a net 
loss of proteoglycan content in 1-year-old animals, but the structure of the joint was maintained. The different effects of Arp2/3 loss in growth plate and articular cartilage is likely reflective of the more permanent nature of articular cartilage as compared with the dynamic and transient cartilage that is found during endochondral ossification and in the growth plate (53).

Noteworthy, Col2-Cre; Arpc2 $2^{\mathrm{fl} / \mathrm{fl}}$ mice presented with an abnormal curvature of the spine that resembled kyphosis. Because Col2a1 expression has been detected in differentiating somites and notochord of the mouse embryo at E9.5 $(34,54)$, Arpc2 deletion in Col2-Cre mice targets sclerotome and notochord cells before the identity of the disc is established. The gross spinal phenotype was associated with impaired ossification of vertebral bodies and severe intervertebral disc defects. The NP compartment was either markedly smaller in size or completely absent due to fusions, while the AF was deformed and contained large chondrocyte-like cells as opposed to cells with a fibroblastic morphology. Previous studies have shown that intervertebral disc fusions can be caused by chondrification of the AF due to abnormal differentiation of AF cells into hypertrophic chondrocytes (55-57). The presence of chondrocyte-like cells rather than fibroblastic-like cells in Arp2/3 mutant mice is consistent with this pathology. Additionally, the fusions might be explained by an impairment of ECM-dependent migration of notochordal cells into the NP compartment or an inability of cells to withstand mechanical forces exerted on the notochord by developing vertebrae during disc development (58). Both of these scenarios are consistent with the central role of Arp2/3 in maintaining actin cytoskeletal integrity, and they explain the severe phenotype of Col2-Cre mutant mice.

In line with our result that Arp2/3 is essential for intervertebral disc health during the early stages of life, Acan-CreER ${ }^{\mathrm{T} 2}$; Arpc2 $2^{\mathrm{f} / \mathrm{fl}}$ mice showed prominent signs of disc degeneration in skeletally mature animals. An interesting effect of induced Arp2/3 loss was an increase in the area of the NP cell band, which was associated with an increase in cell size and number. Previous in vitro studies have shown that Arp2/3-depleted cells have deficits in their ability to decrease their volume under hyperosmotic conditions (5). Therefore, the increase in cell size might be explained by a compromised ability of NP cells to decrease their volume in the proteoglycan-rich and hyperosmotic environment of the disc (23). Though there was no difference in TUNEL ${ }^{+}$cells in mutant mice at 1 year, it is likely that Arpc2 loss may have slowed the age-dependent decline of NP cell number (59). Although less likely, the difference in cell number could also be due to an increase in NP cell proliferation, as it has been shown that proliferation of chondrogenic ATDC5 cells is inhibited by Rac1 or Cdc42 overexpression (13). In conjunction with our findings that the morphology of the NP compartment is severely affected in Col2-Cre; Arpc2 $2^{\mathrm{fl} / \mathrm{fl}}$ mice, the moderate effect of Arp2/3 loss in NP cells of adult Acan-CreER ${ }^{\mathrm{T} 2}$; Arpc2 ${ }^{\mathrm{fl} / \mathrm{ll}}$ mice suggests that Arp2/3 plays a more critical role in early development of this disc compartment. On the other hand, Acan-CreER ${ }^{\mathrm{T} 2}$; Arpc $2^{\mathrm{f} / \mathrm{fl}}$ mice demonstrated pronounced AF degeneration, characterized by loss of demarcation between the NP and AF compartments, lamellae disorganization, and higher incidence of spontaneous annular tears. The susceptibility to spontaneous annular tears was likely due to AF deterioration and altered matrix composition, as mutant animals showed prominent fiber disorganization and buckling, increased collagen degradation as measured by collagen hybridization peptide, reduced abundance levels of collagen IX and COMP, and increased levels of collagen X and the aggrecan degradation product ARGxx $(30,60)$.

Further mechanistic insights into these skeletal phenotypes were provided by our finding that Arp2/3 controls the activity of TonEBP. NP cells showed markedly reduced levels of TauT in Acan-CreER ${ }^{\mathrm{T} 2}$; Arpc $2^{\mathrm{f} / \mathrm{fl}}$ mice and demonstrated decreased levels of TonEBP protein with Arp2/3 inhibition in vitro. The TonEBP transcription factor is expressed by NP cells and chondrocytes to maintain intracellular osmotic balance under hyperosmotic conditions, which are created by an abundance of sulfated proteoglycans and other hydrophilic matrix proteins $(22,49,61)$. Previous reports have shown that hypertonicity activates Rac1 and Cdc42, as well as induces Arp2/3 translocalization to the cell periphery (21). Using HEK293 cells and mouse embryonic fibroblasts, Zhou et al. have also shown that Rac1 mediates TonEBP activity under hyperosmotic conditions via osmosensing scaffold for MEKK3 (OSM) and PLC-y1 (45). More recently, pulldown experiments revealed that Arp2/3 physically interacts with OSM (5). These studies led us to hypothesize that Arp2/3 mediates regulatory effects of Cdc42 and/or Rac1 on hypertonicity-induced activation of TonEBP in cartilage and discs. Indeed, inhibition of Cdc42 or Arp2/3 repressed NaCl-induced TonEBP-TAD reporter activity, as well as the promoter activities and mRNA expression of well-characterized TonEBP target genes, lending the view that TonEBP activity is positively regulated by Cdc42 and Arp2/3. Contrary to findings by Zhou et al., however, TonEBP activity was not affected by inhibition of Rac1-GEF interaction or PLC (45). It should be noted that Rac1-mediated processes may have played a role in driving the various phenotypes observed, 
especially the disorganization seen in the tibial growth plate. Indeed, the chondrodysplastic phenotype of Rac1 ablation in mice is similar to that of the Col2-Cre; Arpc2 $2^{\mathrm{f} / \mathrm{fl}}$ animals characterized herein (10). These studies do, however, indicate that the Rac1-Arp2/3-PLC axis is not involved in the regulation of TonEBP in NP cells, suggesting a cell type-dependent osmoadaptive response. Additionally, since it is known that p38 regulates TonEBP activity in NP cells (26), decreased hypertonic activation of p38 by Arp2/3 inhibition suggests that p38 may contribute to this Cdc42-Arp2/3-TonEBP axis. Taking all of our results into consideration, it is plausible that the observed enlargement of cell size in both NP and growth plate cells lacking Arp2/3 was attributed to an impaired osmotic stress response.

In summary, we conclude that the Arp2/3 complex is critical for cell-ECM interactions, matrix homeostasis, and TonEBP-dependent osmoadaptation in the intervertebral disc and cartilage. This study therefore expands our understanding of the role of Arp2/3 in cartilaginous tissue physiology, with important implications in intervertebral disc degeneration and osteoarthritis (OA), as well as growth plate cartilage defects that contribute to several pathologies in the pediatric population.

\section{Methods}

Mice. The characterization of the conditional Arpc2 allele is provided in a previous publication (33). Other mice used are available from The Jackson Laboratory and have been previously character-

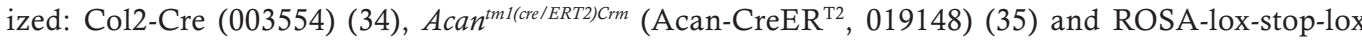
ZsGreen reporter (stock no. 007906) (62). All mice were on a C57BL/6J background, and all analyzed adult mice were males. Embryonic and early postnatal mice were of both sexes. All control animals lacked homozygosity for the Arpc2 transgene and were injected with tamoxifen at the same time points as mutant mice.

Histological analysis. Hindlimbs were fixed in 4\% paraformaldehyde (PFA) for 4 days at $4^{\circ} \mathrm{C}$ and then processed for histological analysis as previously described (63). H\&E staining was used for growth plate organization. In development studies, the number of proliferative columns was assessed by counting instances of 3 or more vertically aligned cells. For analysis of OA in adult hind limbs, Safranin O and Articular Cartilage Scores were graded by a blinded observer according to previously established systems (36). In both grading systems, scores were summed across 4 quadrants (lateral and medial sides of the femoral and tibial surfaces) that were each graded on a 12-point scale, with a higher score indicative of higher OA. Spines were fixed in $4 \%$ PFA for 2 days at $4^{\circ} \mathrm{C}$ and then decalcified in $20 \%$ EDTA at $4^{\circ} \mathrm{C}$ for 14 days. Spines were embedded in paraffin for sectioning in the coronal plane. Sections $(7 \mu \mathrm{m})$ were stained with $1 \%$ Safranin O, 0.05\% Fast Green, and 1\% Hematoxylin and then visualized by light microscopy (Axio Imager 2, Carl Zeiss) using 5×/0.15 N-Achroplan or 20×/0.5 EC Plan-Neofluar (Carl Zeiss) objectives. Imaging of sections was conducted with the Axiocam 105 color camera (Carl Zeiss) using Zen2 software (Carl Zeiss). Disc degeneration was evaluated by 5 blinded observers using a modified Thompson grading scale $(37,38)$. Lumbar levels from seven 6-month-old $(n=28$ discs) and six 1-yearold mutant mice ( $n=24$ discs) were scored. Caudal levels from seven 6-month-old ( $n=27$ discs) and three 1-year-old mutant mice ( $n=11$ discs) were scored. Picrosirius red staining was visualized using a polarized microscope (Eclipse LV100 POL, Nikon). The AF compartments of stained sections were delineated and quantified by percent green, yellow, or red pixels.

$I H C$. Coronal sections of the intervertebral disc were deparaffinized in histoclear and rehydrated in a series of ethanol solutions (100\%-70\%). Deparaffinized sections were incubated in boiled citrate-based unmasking solution (Vector Laboratories, H-3301) for 20 minutes and then cooled to room temperature for 30 minutes. Next, the sections were incubated for 1 hour in the appropriate blocking solution (either 5\%-10\% Normal Goat Serum, 10\% FBS, or reagent from M.O.M. Immunodetection Kit; Vector Laboratories, BMK-2202) and subsequently incubated overnight at $4^{\circ} \mathrm{C}$ with primary antibody against collagen I (1:100; Abcam, ab34710), collagen II (1:400; Fitzgerald, 70R-CR008), COMP (1:200; Abcam, ab231977), collagen IX (1:50; Abcam, ab134568), collagen X (1:500; Abcam, ab58632), ARGxx (1:200; Abcam, ab3773), CA3 (1:150; Santa Cruz Biotechnology Inc., sc-50715), keratin-19 (1:50; Developmental Studies Hybridoma Bank [DSHB] TROMA-III/supernatant), vimentin (1:200; Cell Signaling Technology, D21H3), aggrecan (1:50; MilliporeSigma, AB1031), chondroitin sulfate (1:300; Abcam, ab11570), TauT (1:100; Abcam, ab196821), TonEBP (1:200; Abcam, ab3446), SOX9 (1:250; Abcam, ab185230), and Ki67 (1:100; Abcam, ab15580). The ARGxx antibody recognizes the ARG neoepitope generated after aggrecanase (ADAMTS-1, -4, and -5) cleavage between amino acids EGE and ARG within aggrecan's interglobular domain. Collagen degradation was analyzed by staining 
with a collagen hybridization peptide, $\mathrm{Cy} 3$ conjugate (R-CHP; 3Helix). After washing with PBS, sections were incubated for 1 hour at room temperature with Alexa Fluor-594 secondary antibody (1:700, Jackson ImmunoResearch). The sections were washed with PBS before mounting with ProLong Gold Antifade Mountant containing DAPI (Thermo Fisher Scientific, P36934) and visualized by fluorescence microscopy (Axio Imager 2, Carl Zeiss) using the $5 \times / 0.15$ N-Achroplan or 20×/0.5 EC Plan-Neofluar (Carl Zeiss) objectives. The stained sections were imaged using X-Cite 120Q Excitation Light Source (Excelitas), the AxioCam MRm camera (Carl Zeiss), and Zen2 software (Carl Zeiss). Five 1-year-old animals per group were used for analysis, and staining was performed on 2 representative lumbar discs per animal ( $n=10$ discs/group).

TUNEL assay. The In Situ Cell Death Detection Kit TMR Red (MilliporeSigma) was used to measure cell death of 1-year-old lumbar discs. Slides were heated at $60^{\circ} \mathrm{C}$ for 3 hours, deparaffinized, rehydrated through a graded series of ethanol solutions, and permeabilized with boiling citrate-based unmasking solution ( $\mathrm{pH}$ 6) (Vector Laboratories, H-3301) for 30 minutes at room temperature. The TUNEL assay was then performed as per manufacturer's protocol. A positive control was generated using RNAse Free DNAse I (QIAGEN). Sections were washed with PBS before mounting with ProLong Gold Antifade Mountant containing DAPI (Thermo Fisher Scientific, P36934) and visualized by fluorescence microscopy (Axio Imager 2, Carl Zeiss) using the 20×/0.5 EC Plan-Neofluar (Carl Zeiss) objective. The stained sections were imaged using X-Cite 120Q Excitation Light Source (Excelitas), the AxioCam MRm camera (Carl Zeiss), and Zen2 software (Carl Zeiss). Staining was performed on 10 discs/group (5 animals/group; 2 discs/animal).

Digital image analysis. All imaged sections stained by IHC were analyzed using ImageJ 1.52a (http://rsb. info.nih.gov/ij; NIH) in the grayscale. The boundaries of the NP and AF were digitally traced using the freehand tool. These images were then thresholded to create binary images. Designated regions of interest (ROI) were analyzed using the area fraction measurement for each section. Area fraction represents the percentage of positive pixels normalized to ROI, representing protein abundance within a given area. Similarly, cell count was measured by performing a watershed and particle analysis on thresholded images of DAPI-stained sections.

Cell spreading assay on FACS-sorted chondrocytes with Arp2/3 inactivation. At P12, the Acan-CreER ${ }^{\mathrm{T} 2}$ allele was activated by a single i.p. injection of tamoxifen. At P19, the hind limb was dissected to separate growth plate and articular cartilage tissues using a scalpel. Each tissue was independently digested with $0.04 \%$ collagenase $\mathrm{P}$ (11 249002 001, MilliporeSigma) overnight under rotation in a tissue culture incubator. After digestion, chondrocytes were filtered through a 30- $\mu$ m strainer to remove undigested clumps of cells, washed with HBSS, and prepared in a single-cell suspension in HBSS with 2\% FBS and 10 mM EDTA for flow cytometry cell sorting. Using a benchtop sorter (Bio-Rad, S3e), chondrocytes were gated based on ZsGreen signal as compared with a WT gating control, and $\mathrm{ZsGreen}^{+}$cells were sorted directly into culture tubes. After sorting, the ZsGreen ${ }^{+}$ growth plate or articular chondrocytes were allowed to spread on either $10 \mu \mathrm{g} / \mathrm{mL}$ collagen or $10 \mu \mathrm{g} / \mathrm{mL}$ fibronectin-coated coverslips and imaged via phase contrast as cells spread overnight. Individual cell outlines in the last frame of each image series were traced by hand in ImageJ to obtain cell spread areas. Cells were then fixed and stained with fluorescent phalloidin.

NP cell isolation and treatment. NP cells were isolated from Sprague-Dawley rats (Charles River Laboratories) as previously described (24). Cells were maintained in DMEM containing $1 \mathrm{~g} / \mathrm{L}$ glucose with $10 \%$ FBS and $1 \%$ penicillin-streptomycin (30-001-CI, Corning). After cells were treated with $100 \mu \mathrm{M}$ of CK666 (182525, MilliporeSigma), $10 \mu \mathrm{M}$ of ML-141 (SML0407, MilliporeSigma), $100 \mu \mathrm{M}$ of NSC23766 (SML0952, MilliporeSigma), or 250/500 $\mu \mathrm{M}$ of U73122 (1268, Tocris) for 1 hour, $110 \mathrm{mM} \mathrm{NaCl}$ was added to medium for 7-hour treatment. NSC23766 inhibits Rac1 activation by blocking its interaction with Rac-specific guanine nucleotide exchange factors (GEFs). For cell spreading assays, $1 \times 10^{5} \mathrm{NP}$ cells were plated on $10 \mu \mathrm{g} / \mathrm{mL}$ collagen type I-coated (MilliporeSigma) or $10 \mu \mathrm{g} / \mathrm{mL}$ fibronectin-coated (MilliporeSigma) coverslips with or without addition of Arp2/3 inhibitor (CK666). Cells were allowed to spread over 14 hours before fixing and labeling with fluorescent phalloidin for actin. Cells were imaged using Zeiss Axio Imager A2 (Carl Zeiss) or Zeiss LSM 800.

Immunofluorescence staining. A total of $2 \times 10^{5}$ rat NP cells were plated on glass coverslips and allowed to spread overnight. Cells were treated with $100 \mu \mathrm{M}$ CK666 1 hour before the addition of media containing 110 $\mathrm{mM} \mathrm{NaCl}$, and they were supplemented with $100 \mu \mathrm{M}$ CK666 for 7 hours. Control cells were cultured under isotonic conditions without an inhibitor. Cells were fixed with 4\% PFA for 10 minutes, permeabilized with 0.1\% Triton X-100 (BP151-100, Thermo Fisher Scientific) in PBS for 5 minutes, and blocked with 1\% BSA in PBS-Tween $0.1 \%$ (1706531, Bio-Rad) for 1 hour at room temperature. Cells were incubated with anti-TonEBP antibody (1:500; Abcam, ab3446) for 1 hour and were then washed before labeling with fluorescent phalloidin, 
Alexa Fluor 488-conjugated secondary antibody. Cells were mounted with DAPI-containing mounting media and imaged using Zeiss scanning confocal (LSM 800 Axiocam 506 mono with a Plan-Apochromat 40×, 1.3 NA oil objective). Brightness and contrast were linearly adjusted using ImageJ 1.52b.

Confocal imaging for quantification of actin abundance. We used confocal microscopy to analyze actin abundance in chondrocytes with and without the loss of Arpc2. Femoral condyles of 3 Acan-CreER ${ }^{\mathrm{T} 2}$; Arpc $2^{\mathrm{f} / / \mathrm{fl}}$; ROSA-lox-stop-lox-ZsGreen mice were assessed, with cartilage containing a mix of cells that had either been recombined by the Cre driver or not. We initiated the loss of Arpc2 in a subset of chondrocytes at 4 months of age by treating mice with a single low dose of tamoxifen. The dose of $2.5 \mu \mathrm{g}$ tamoxifen per gram mouse weight was $1 / 10$ the dose used for near-complete recombination in other experiments of this study. At 6 months of age, mice were sacrificed, and the femoral condyle was dissected. After fixation with $4 \%$ PFA, the tissue was stained with phalloidin 647 (A22287, Thermo Fisher Scientific) and DAPI for 1 hour, washed 3 times, and then positioned in a Mat-Tek chamber using molten agarose. $Z$ stacks were obtained on an inverted Fluoview FV1000 scanning confocal microscope (Olympus) using a 40× objective. The maximum intensity projection of $8 \times 4 \mu \mathrm{m}$ stacks was used to minimize lost staining due to the actin cortex being out of the focal plane. Image was used to quantify the integrated pixel intensity of 78 Arpc2-intact cells (no recombination as denoted by lack of a ZsGreen signal in that cell) and 69 Arpc2-loss cells (recombination as denoted by a positive ZsGreen signal in that cell). The cells were quantified from across femoral condyles of 3 mice/group. Note that, for ease of viewing, the ZsGreen signal is pseudocolored red and the phalloidin signal is pseudocolored green.

Plasmids. Luciferase reporter plasmids were provided by Takashi Ito (Osaka University, Suita, Japan) (TauT Wt; Mt) (64), H. M. Kwon (Ulsan National Institute of Science and Technology, Ulsan, South Korea) (Hsp-70 Wt; Mt) (65), and Ferraris and coworkers (NIH) (Ton-TAD, pFR-luc) (66). AR luc was from Addgene (14110). pRL-TK (Promega) was used as a transfection control.

Transfections and dual-luciferaseassay. Cells were transferred to 48-well plates (2104 cells/well) 1 day before transfection. To measure the effects of stimuli on TauT-luc activity, cells were transfected with 250 ng of TauT promoter reporter and 250 ng of pRL-TK plasmid and cultured in control, hypertonic, CK666, ML-141, NSC23766, or U73122 treatment conditions. In all experiments, plasmids were premixed with the transfection reagent, Lipofectamine 2000 (Invitrogen). Forty-eight hours after transfection, cells were harvested, and firefly and Renilla luciferase activities were measured using the Dual-Luciferase reporter assay (Promega) and a luminometer (Tecan Infinite M200). Data were collected from 3 independent biological experiments with 3 technical replicates.

Quantitative PCR. Total RNA was extracted from primary NP cells using RNeasy mini columns (Qiagen) and RNAse free DNAse I (Qiagen). The eluted DNA-free RNA was made into cDNA using EcoDry premix (Clontech). PCR reactions using gene-specific primers (Integrated DNA Technologies) and SYBR Green master mix (Applied Biosystems) were measured by the Step-One Plus System (Applied Biosystems). Experiments were conducted with at least 4 biological replicates.

Western blot. Rat NP cells $\left(4 \times 10^{5}\right.$ cells / plate) were plated on $6-\mathrm{cm}$ dishes and treated with or without CK666 $(100 \mu \mathrm{M})$ under isotonic or hypertonic conditions $(110 \mathrm{mM} \mathrm{NaCl})$ for 8 hours. Cells were lysed in $1 \times$ protease inhibitor-containing lysis buffer. Total protein was resolved on $8 \%$ SDS-PAGE and transferred onto PVDF membranes. Membranes were blocked with 5\% nonfat dry milk in TBS with $0.1 \%$ Tween-20 for 1 hour at room temperature and incubated overnight at $4{ }^{\circ} \mathrm{C}$ in $3 \%$ to $5 \%$ nonfat dry milk in TBST with antibodies against TonEBP (1:1000; Abcam, ab3446) or GAPDH (1:3000; Cell Signaling Technologies, 14C10). Immunolabeling was detected using ECL (Amersham Biosciences), and densitometric quantification was performed using ImageJ software.

In-cell Western assay. A total of $1 \times 10^{5}$ Rat NP cells/well were plated on 96-well plates (CellStar, 655090) and allowed to proliferate for 2 days. Cells were treated with or without CK666 $(100 \mu \mathrm{M})$ in the presence or absence of hypertonic media $(110 \mathrm{mM} \mathrm{NaCl})$ for 15 minutes to 4 hours. Cells were fixed with $3.7 \%$ PFA for 10 minutes, permeabilized with $0.1 \%$ Triton X-100 in PBS for 5 minutes, and blocked with Odyssey Blocking Buffers (LI-COR, 927-50000) for 1 hour at room temperature. Cells were then probed with antibodies diluted in Odyssey Blocking Buffers against Erk, phosphorylated Erk, p38, or phosphorylated p38 for 2.5 hours at room temperature (Cell Signaling Technologies; 4696S, 9101S, 9212, 9211S, respectively; 1:250). Cells were then labeled with appropriate secondary antibodies or CellTag 700 stain for 1 hour in the absence of light (LI-COR; goat anti-rabbit IgG secondary antibody, 926-32211; goat anti-mouse IgG secondary antibody, 926-68070; CellTa 700 Stain for In-Cell Wester Assays, 926-41090; 1:1000). Cells were washed, and plates were scanned using Odyssey CLx Infrared Imaging System (LI-COR). Total intensities per channel were acquired using the 
software provided with the imager station (Odyssey Software Version 3.0, LI-COR). The relative intensity of the signal was obtained by phosphorylated protein intensity normalized to total protein intensity.

Statistics. Data are presented as mean \pm SD. To determine significance, 2-tailed, unpaired Student's $t$ test or 1-way ANOVA were used for continuous variables with normal distributions, whereas the Mann-Whitney $U$ or Kruskal-Wallis tests were used when data were not normally distributed, as determined by the Shapiro-Wilk test. Significance between collagen fiber distributions was determined by $\chi^{2}$ test. Since the unique interactions between genetic, biological, and biomechanical factors at individual spinal levels have been shown to produce different phenotypic outcomes, each disc was considered as an independent sample (30, 40-42). All statistical analyses were done using Prism 7 (GraphPad Software). $P \leq 0.05$ was the threshold for statistical significance.

Study approval. All mouse experiments were performed under protocols approved by the IACUC of the University of North Carolina at Chapel Hill. Collection of rat NP tissue was performed under protocols approved by IACUC of the Thomas Jefferson University, in accordance with the IACUC's relevant guidelines and regulations.

\section{Author contributions}

MVR, BOD, ST, JDR, JEB, VU, and RFL designed the study. MVR, IMS, BOD, JDR, and VU conducted the study. BOD, ST, ACD, JDR, VU, and KS collected data. BOD, ST, ACD, JDR, VU, and KS analyzed data. MVR, BOD, ST, JDR, JEB, RFL, and KS interpreted data. MVR, BOD, and ST drafted the manuscript. MVR, IMS, BOD, ST, JDR, and VU revised the manuscript. MVR, IMS, BOD, ST, ACD, JDR, $\mathrm{JEB}, \mathrm{VU}$, and RFL approved the final version of the manuscript.

\section{Acknowledgments}

The authors thank Norman Sharpless of the University of North Carolina at Chapel Hill for contributions to the early phases of this work. This work was supported by the NIH under grant numbers R01AR064733, R01AR055655, R01AR074813, T32AR052273, R37AR049003, and R35GM130312. This work was also supported by the NIH F32AG050399 (BOD) and was conducted while BOD was an Arthritis and Aging Research Grant recipient from the Arthritis National Research Foundation (ANRF) and the American Federation for Aging Research; this work also received second-year funding from ANRF.

Address correspondence to: Makarand V. Risbud, Department of Orthopaedic Surgery, Thomas Jefferson University, 1025 Walnut Street, Suite 501 College Building, Philadelphia Pennsylvania 19107, USA. Phone: 215.955.1063; Email: makarand.risbud@jefferson.edu. Or to: Brian O. Diekman, Thurston Arthritis Research Center, 3300 Thurston Building, Campus box \#7280, University of North Carolina at Chapel Hill,, Chapel Hill, North Carolina 27599-7280, USA. Phone: 919.445.2613; Email: bdiekman@email.unc.edu.

1. Abella JV, et al. Isoform diversity in the Arp2/3 complex determines actin filament dynamics. Nat Cell Biol. 2016;18(1):76-86.

2. Robinson RC, et al. Crystal structure of Arp2/3 complex. Science. 2001;294(5547):1679-1684.

3. Rotty JD, Wu C, Bear JE. New insights into the regulation and cellular functions of the ARP2/3 complex. Nat Rev Mol Cell Biol. 2013;14(1):7-12.

4. Pizarro-Cerdá J, Chorev DS, Geiger B, Cossart P. The Diverse Family of Arp2/3 Complexes. Trends Cell Biol. 2017;27(2):93-100.

5. Wu C, et al. Loss of Arp2/3 induces an NF-кB-dependent, nonautonomous effect on chemotactic signaling. J Cell Biol. 2013;203(6):907-916

6. Wu C, et al. Arp2/3 is critical for lamellipodia and response to extracellular matrix cues but is dispensable for chemotaxis. Cell. 2012;148(5):973-987.

7. Rohatgi R, et al. The interaction between N-WASP and the Arp2/3 complex links Cdc42-dependent signals to actin assembly. Cell. 1999;97(2):221-231.

8. Miki H, Suetsugu S, Takenawa T. WAVE, a novel WASP-family protein involved in actin reorganization induced by Rac. $E M B O$ J. 1998;17(23):6932-6941.

9. Machesky LM, Insall RH. Scar1 and the related Wiskott-Aldrich syndrome protein, WASP, regulate the actin cytoskeleton through the Arp2/3 complex. Curr Biol. 1998;8(25):1347-1356.

10. Wang G, Woods A, Agoston H, Ulici V, Glogauer M, Beier F. Genetic ablation of Rac1 in cartilage results in chondrodysplasia. Dev Biol. 2007;306(2):612-623.

11. Nagahama R, et al. Rho GTPase protein Cdc42 is critical for postnatal cartilage development. Biochem Biophys Res Commun. 2016;470(4):813-817.

12. Aizawa R, et al. Cdc42 is required for chondrogenesis and interdigital programmed cell death during limb development. Mech Dev. 2012;129(1-4):38-50.

13. Wang G, Beier F. Rac1/Cdc42 and RhoA GTPases antagonistically regulate chondrocyte proliferation, hypertrophy, and apoptosis. J Bone Miner Res. 2005;20(6):1022-1031. 
14. Aszodi A, Hunziker EB, Brakebusch C, Fässler R. Beta1 integrins regulate chondrocyte rotation, G1 progression, and cytokinesis. Genes Dev. 2003;17(19):2465-2479.

15. Grashoff C, Aszódi A, Sakai T, Hunziker EB, Fässler R. Integrin-linked kinase regulates chondrocyte shape and proliferation. EMBO Rep. 2003;4(4):432-438.

16. Terpstra L, et al. Reduced chondrocyte proliferation and chondrodysplasia in mice lacking the integrin-linked kinase in chondrocytes. J Cell Biol. 2003;162(1):139-148.

17. Raducanu A, Hunziker EB, Drosse I, Aszódi A. Beta1 integrin deficiency results in multiple abnormalities of the knee joint. J Biol Chem. 2009;284(35):23780-23792.

18. Humphries JD, et al. Proteomic analysis of integrin-associated complexes identifies RCC2 as a dual regulator of Rac1 and Arf6. Sci Signal. 2009;2(87):ra51.

19. Chorev DS, Moscovitz O, Geiger B, Sharon M. Regulation of focal adhesion formation by a vinculin-Arp2/3 hybrid complex. Nat Commun. 2014;5:3758.

20. DeMali KA, Barlow CA, Burridge K. Recruitment of the Arp2/3 complex to vinculin: coupling membrane protrusion to matrix adhesion. J Cell Biol. 2002;159(5):881-891.

21. Di Ciano C, et al. Osmotic stress-induced remodeling of the cortical cytoskeleton. Am J Physiol, Cell Physiol. 2002;283(3):C850-C865

22. Silagi ES, Shapiro IM, Risbud MV. Glycosaminoglycan synthesis in the nucleus pulposus: Dysregulation and the pathogenesis of disc degeneration. Matrix Biol. 2018;71-72:368-379.

23. Johnson ZI, Shapiro IM, Risbud MV. Extracellular osmolarity regulates matrix homeostasis in the intervertebral disc and articular cartilage: evolving role of TonEBP. Matrix Biol. 2014;40:10-16.

24. Tsai TT, et al. TonEBP/OREBP is a regulator of nucleus pulposus cell function and survival in the intervertebral disc. $J$ Biol Chem. 2006;281(35):25416-25424.

25. van der Windt AE, et al. Physiological tonicity improves human chondrogenic marker expression through nuclear factor of activated T-cells 5 in vitro. Arthritis Res Ther. 2010;12(3):R100.

26. Tsai TT, Guttapalli A, Agrawal A, Albert TJ, Shapiro IM, Risbud MV. MEK/ERK signaling controls osmoregulation of nucleus pulposus cells of the intervertebral disc by transactivation of TonEBP/OREBP. J Bone Miner Res. 2007;22(7):965-974.

27. Choi H, et al. COX-2 expression mediated by calcium-TonEBP signaling axis under hyperosmotic conditions serves osmoprotective function in nucleus pulposus cells. J Biol Chem. 2018;293(23):8969-8981.

28. Burg MB, Ferraris JD. Intracellular organic osmolytes: function and regulation. J Biol Chem. 2008;283(12):7309-7313.

29. Tessier S, Madhu V, Johnson ZI, Shapiro IM, Risbud MV. NFAT5/TonEBP controls early acquisition of notochord phenotypic markers, collagen composition, and sonic hedgehog signaling during mouse intervertebral disc embryogenesis. Dev Biol. 2019;455(2):369-381.

30. Tessier S et al. TonEBP-deficiency accelerates intervertebral disc degeneration underscored by matrix remodeling, cytoskeletal rearrangements, and changes in proinflammatory gene expression [published online November 9, 2019]. Matrix Biol. https://doi.org/10.1016/j.matbio.2019.10.007.

31. Stradal TE, Rottner K, Disanza A, Confalonieri S, Innocenti M, Scita G. Regulation of actin dynamics by WASP and WAVE family proteins. Trends Cell Biol. 2004;14(6):303-311.

32. Goley ED, Rammohan A, Znameroski EA, Firat-Karalar EN, Sept D, Welch MD. An actin-filament-binding interface on the Arp2/3 complex is critical for nucleation and branch stability. Proc Natl Acad Sci USA. 2010;107(18):8159-8164.

33. Rotty JD, et al. Profilin-1 serves as a gatekeeper for actin assembly by Arp2/3-dependent and -independent pathways. Dev Cell. 2015;32(1):54-67.

34. Ovchinnikov DA, Deng JM, Ogunrinu G, Behringer RR. Col2a1-directed expression of Cre recombinase in differentiating chondrocytes in transgenic mice. Genesis. 2000;26(2):145-146.

35. Henry SP, Jang CW, Deng JM, Zhang Z, Behringer RR, de Crombrugghe B. Generation of aggrecan-CreERT2 knockin mice for inducible Cre activity in adult cartilage. Genesis. 2009;47(12):805-814.

36. McNulty MA, Loeser RF, Davey C, Callahan MF, Ferguson CM, Carlson CS. A Comprehensive Histological Assessment of Osteoarthritis Lesions in Mice. Cartilage. 2011;2(4):354-363.

37. Choi $\mathrm{H}$, et al. A novel mouse model of intervertebral disc degeneration shows altered cell fate and matrix homeostasis. Matrix Biol. 2018;70:102-122.

38. Thompson JP, Pearce RH, Schechter MT, Adams ME, Tsang IK, Bishop PB. Preliminary evaluation of a scheme for grading the gross morphology of the human intervertebral disc. Spine. 1990;15(5):411-415.

39. Muriuki MG, et al. Effects of motion segment level, Pfirrmann intervertebral disc degeneration grade and gender on lumbar spine kinematics. J Orthop Res. 2016;34(8):1389-1398.

40. Battié MC, Videman T, Levälahti E, Gill K, Kaprio J. Genetic and environmental effects on disc degeneration by phenotype and spinal level: a multivariate twin study. Spine. 2008;33(25):2801-2808.

41. Li Y, et al. Two subtypes of intervertebral disc degeneration distinguished by large-scale population-based study. Spine J. 2016;16(9):1079-1089.

42. Vincent K, et al. Aging of mouse intervertebral disc and association with back pain. Bone. 2019;123:246-259.

43. Halász K, Kassner A, Mörgelin M, Heinegård D. COMP acts as a catalyst in collagen fibrillogenesis. J Biol Chem. 2007;282(43):31166-31173.

44. Novais EJ, Diekman BO, Shapiro IM, Risbud MV. p16 ${ }^{\text {Ink4a }}$ deletion in cells of the intervertebral disc affects their matrix homeostasis and senescence associated secretory phenotype without altering onset of senescence. Matrix Biol. 2019;82:54-70.

45. Zhou X, Izumi Y, Burg MB, Ferraris JD. Rac1/osmosensing scaffold for MEKK3 contributes via phospholipase C-gamma1 to activation of the osmoprotective transcription factor NFAT5. Proc Natl Acad Sci USA. 2011;108(29):12155-12160.

46. Di Ciano-Oliveira C, Thirone AC, Szászi K, Kapus A. Osmotic stress and the cytoskeleton: the R(h)ole of Rho GTPases. Acta Physiol (Oxf). 2006;187(1-2):257-272.

47. Lewis A, Di Ciano C, Rotstein OD, Kapus A. Osmotic stress activates Rac and Cdc42 in neutrophils: role in hypertonicity-induced actin polymerization. Am J Physiol, Cell Physiol. 2002;282(2):C271-C279.

48. van Dijk B, Potier E, Ito K. Culturing bovine nucleus pulposus explants by balancing medium osmolarity. Tissue Eng Part C 
Methods. 2011;17(11):1089-1096.

49. Ishihara H, Warensjo K, Roberts S, Urban JP. Proteoglycan synthesis in the intervertebral disk nucleus: the role of extracellular osmolality. Am J Physiol. 1997;272(5 Pt 1):C1499-C1506.

50. Roth I, Leroy V, Kwon HM, Martin PY, Féraille E, Hasler U. Osmoprotective transcription factor NFAT5/TonEBP modulates nuclear factor-kappaB activity. Mol Biol Cell. 2010;21(19):3459-3474.

51. Ikehata M, et al. Cooperation of Rho family proteins Rac1 and Cdc42 in cartilage development and calcified tissue formation. Biochem Biophys Res Commun. 2018;500(3):525-529.

52. Dy P, et al. Sox 9 directs hypertrophic maturation and blocks osteoblast differentiation of growth plate chondrocytes. Dev Cell. 2012;22(3):597-609.

53. Decker RS, Koyama E, Pacifici M. Articular Cartilage: Structural and Developmental Intricacies and Questions. Curr Osteoporos Rep. 2015;13(6):407-414.

54. Wood A, Ashhurst DE, Corbett A, Thorogood P. The transient expression of type II collagen at tissue interfaces during mammalian craniofacial development. Development. 1991;111(4):955-968.

55. Zieba J, et al. TGF $\beta$ and BMP Dependent Cell Fate Changes Due to Loss of Filamin B Produces Disc Degeneration and Progressive Vertebral Fusions. PLoS Genet. 2016;12(3):e1005936.

56. Ulici V, et al. Impaired Annulus Fibrosus Development and Vertebral Fusion Cause Severe Scoliosis in Mice with Deficiency of c-Jun NH2-Terminal Kinases 1 and 2. Am J Pathol. 2019;189(4):868-885.

57. Tanaka T, Uhthoff HK. The pathogenesis of congenital vertebral malformations. A study based on observations made in 11 human embryos and fetuses. Acta Orthop Scand. 1981;52(4):413-425.

58. Lawson L, Harfe BD. Notochord to Nucleus Pulposus Transition. Curr Osteoporos Rep. 2015;13(5):336-341.

59. Dahia CL, Mahoney EJ, Durrani AA, Wylie C. Postnatal growth, differentiation, and aging of the mouse intervertebral disc. Spine. 2009;34(5):447-455

60. Gorth DJ, Ottone OK, Shapiro IM, Risbud MV. Differential effect of long-term systemic exposure of TNF $\alpha$ on health of the annulus fibrosus and nucleus pulposus of the intervertebral disc [published online December 4, 2019]. J Bone Miner Res. https://doi.org/10.1002/jbmr.3931

61. Urban JP. The chondrocyte: a cell under pressure. Br J Rheumatol. 1994;33(10):901-908.

62. Madisen L, et al. A robust and high-throughput Cre reporting and characterization system for the whole mouse brain. Nat Neurosci. 2010;13(1):133-140.

63. Sessions GA, Copp ME, Liu JY, Sinkler MA, D'Costa S, Diekman BO. Controlled induction and targeted elimination of p16 $6^{\text {INK4a }}$-expressing chondrocytes in cartilage explant culture. FASEB J. 2019;33(11):12364-12373.

64. Ito T, et al. Expression of taurine transporter is regulated through the TonE (tonicity-responsive element)/TonEBP (TonE-binding protein) pathway and contributes to cytoprotection in HepG2 cells. Biochem J. 2004;177-182.

65. Woo SK, et al. Bidirectional regulation of tonicity-responsive enhancer binding protein in response to changes in tonicity. Am J Physiol Renal Physiol. 2000;F1006-F1012.

66. Ferraris JD, et al. Activity of the TonEBP/OREBP transactivation domain varies directly with extracellular NaCl concentration. Proc Natl Acad Sci USA. 2002;739-744. 